TRANSACTIONS OF THE

AMERICAN MATHEMATICAL SOCIETY

Volume 359, Number 9, September 2007, Pages 4359-4387

S 0002-9947(07)04142-6

Article electronically published on March 20, 2007

\title{
BASES FOR SOME RECIPROCITY ALGEBRAS I
}

\author{
ROGER HOWE AND SOO TECK LEE
}

\begin{abstract}
For a complex vector space $V$, let $\mathcal{P}(V)$ be the algebra of polynomial functions on $V$. In this paper, we construct bases for the algebra of all $\mathrm{GL}_{n}(\mathbb{C}) \times \mathrm{GL}_{m_{1}}(\mathbb{C}) \times \mathrm{GL}_{m_{2}}(\mathbb{C}) \times \cdots \times \mathrm{GL}_{m_{r}}(\mathbb{C})$ highest weight vectors in $\mathcal{P}\left(\mathbb{C}^{n} \otimes \mathbb{C}^{m}\right)$, where $m=m_{1}+\cdots+m_{r}$ and $m_{j} \leq n$ for all $1 \leq j \leq r$, and the algebra of $\mathrm{GL}_{n}(\mathbb{C}) \times \mathrm{GL}_{k}(\mathbb{C}) \times \mathrm{GL}_{1}(\mathbb{C})$ highest weight vectors in $\mathcal{P}\left[\left(\mathbb{C}^{n} \otimes \mathbb{C}^{k}\right) \oplus\left(\mathbb{C}^{n *} \otimes \mathbb{C}^{l}\right)\right]$.
\end{abstract}

\section{INTRODUCTION}

This paper continues the study of branching laws for symmetric subgroups of classical groups begun in HTW1, HTW2, HTW3. Those papers construct and study certain algebras, called branching algebras, which describe the branching laws in question. In a sense made precise in HTW1, the most fundamental of these algebras is the $\mathrm{GL}_{n}$ tensor product algebra, which describes the decomposition of tensor products of representations of the general linear group $\mathrm{GL}_{n}=\mathrm{GL}_{n}(\mathbb{C})$. The paper HTW3 describes a basis for the $\mathrm{GL}_{n}$ tensor product algebra. The basis elements constructed in HTW3 are parametrized in a fairly straightforward way by the Littlewood-Richardson tableaux ([Ful]).

This paper begins a project of adapting and extending the construction of HTW3 to other branching algebras. Specifically, here we describe bases for two variants of the $\mathrm{GL}_{n}$ tensor product algebra. The specific algebras considered here are the iterated tensor product algebra for $\mathrm{GL}_{n}$ and also, a variant of the usual tensor product algebra, in which one takes tensor products of polynomial representations with duals of polynomial representations. These are the simplest cases beyond the $\mathrm{GL}_{n}$ tensor product algebra, and they will be useful for dealing with more complicated constructions, so it seems worthwhile to present them separately here.

For the construction of [HTW3], the $\mathrm{GL}_{n}$ tensor product algebra is realized as an explicit algebra of polynomials on a space of matrices, and each basis element is a specific polynomial. The key property of the construction is that, with respect to a certain monomial order, each basis element has a highest term which allows one to reconstruct the tableau from which it came. This shows in particular that the basis constructed is a SAGBI basis ( $[\mathrm{RS}$, , $\mathrm{Stu}]$ ) for the $\mathrm{GL}_{n}$ tensor product algebra. It also enables one to show that the $\mathrm{GL}_{n}$ tensor product algebra is a flat deformation of the semigroup ring attached to the Berenstein-Zelevinsky cone ([BZ, Ho95]). In particular, it is a flat deformation of a toric variety. One main goal of our constructions in this paper and its successors is to lay the groundwork

Received by the editors April 8, 2005 and, in revised form, August 22, 2005.

2000 Mathematics Subject Classification. Primary 22E46. 
for such a result for other families of branching algebras for classical symmetric pairs ([HTW1]).

Recently, a number of authors ( $\mathrm{GL}, \mathrm{Ch}, \mathrm{Ca}, \mathrm{KM}]$ ) have shown that the coordinate ring of (a torus bundle over) a flag variety, or of a more general spherical variety, can be deformed to a toric variety. This may be viewed as an abstract version of the Standard Monomial Theory ([Hd], [DEP, [LMS1], LMS2, [L1], L2, L3, L4, LLM], LS, $\mathrm{BL})$. Our results are in the same spirit. However, the branching algebras we are dealing with are substantially more complicated than coordinate rings of spherical varieties, and the cones associated to the toric varieties will correspondingly be more complicated. Whereas generators and relations for the coordinate ring of the flag manifold for $\mathrm{GL}_{n}$ have been known since the time of Hodge $([\mathrm{Hd}])$, generators and relations for branching algebras are known in only a very few cases. Describing these algebras as deformations of semigroup rings should provide greater insight into such issues.

We now describe the two algebras which we shall study in this paper. For each positive integer $m$, let $U_{m}$ be the standard maximal unipotent subgroup of $\mathrm{GL}_{m}$ consisting of all $m \times m$ upper triangular matrices with 1's on the diagonal. The first algebra is the algebra

$$
\mathrm{TA}_{n, l_{1}, \ldots, l_{k}}=\mathcal{P}\left(\mathbb{C}^{n} \otimes \mathbb{C}^{l}\right)^{U_{n} \times\left(U_{l_{1}} \times \cdots \times U_{l_{k}}\right)}
$$

of all $\mathrm{GL}_{n} \times\left(\mathrm{GL}_{l_{1}} \times \cdots \times \mathrm{GL}_{l_{k}}\right)$ highest weight vectors in $\mathcal{P}\left(\mathbb{C}^{n} \otimes \mathbb{C}^{l}\right)$, where $l_{j} \leq n$ for all $1 \leq j \leq k$ and $l=l_{1}+\cdots+l_{k}$. It describes the decomposition of $k$-fold tensor products of irreducible $\mathrm{GL}_{n}$ representations into sums of irreducible $\mathrm{GL}_{n}$ representations. Therefore we shall call it a $k$-fold tensor product algebra for $\mathrm{GL}_{n}$. On the other hand, it also describes the decomposition of $\mathrm{GL}_{l}$ irreducible representations into sums of $\mathrm{GL}_{l_{1}} \times \cdots \times \mathrm{GL}_{l_{k}}$ irreducible representations. Since $\mathrm{TA}_{n, l_{1}, \ldots, l_{k}}$ carries two kinds of branching information associated with a see-saw dual pair, it is a reciprocity algebra in the sense of HTW1. We will construct a basis for $\mathrm{TA}_{n, l_{1}, \ldots, l_{k}}$ which is parametrized by a set of $(k-1)$-th tuples $\left(T_{1}, \ldots, T_{k-1}\right)$ of Littlewood-Richardson tableaux. (Strictly speaking, the tableaux should be embedded in a nested sequence $F_{1} \subseteq F_{2} \subseteq \cdots \subseteq F_{k-1}$ of Young diagrams.)

Let $n, k$ and $l$ be positive integers with $n \geq k+l$, and let $V_{n, k, l}$ be the $\mathrm{GL}_{n} \times$ $\mathrm{GL}_{k} \times \mathrm{GL}_{l}$ module

$$
V_{n, k, l}=\left(\mathbb{C}^{n} \otimes \mathbb{C}^{k}\right) \oplus\left(\mathbb{C}^{n *} \otimes \mathbb{C}^{l}\right) .
$$

The second algebra is the algebra $\mathcal{P}\left(V_{n, k, l}\right)^{U_{n} \times U_{k} \times U_{l}}$ of $\mathrm{GL}_{n} \times \mathrm{GL}_{k} \times \mathrm{GL}_{l}$ highest weight vectors in $\mathcal{P}\left(V_{n, k, l}\right)$. We will construct a basis for this algebra which is parametrized by a set of ordered pairs $\left(T_{1}, T_{2}\right)$ of Littlewood-Richardson tableaux. The algebra $\mathcal{P}\left(V_{n, k, l}\right)^{U_{n} \times U_{k} \times U_{l}}$ is also a reciprocity algebra. For any Young diagram $A$ with at most $m$ rows, we denote the corresponding irreducible representation of $\mathrm{GL}_{m}$ by $\rho_{m}^{A}$, and the dual representation of $\rho_{m}^{A}$ by $\rho_{m}^{A^{*}}$. Then from the basis for $\mathcal{P}\left(V_{n, k, l}\right)^{U_{n} \times U_{k} \times U_{l}}$, we obtain a basis for the space of $\mathrm{GL}_{n}$ highest weight vectors in any tensor product of $\mathrm{GL}_{n}$ modules of the form $\rho_{n}^{G} \otimes \rho_{n}^{H^{*}}$, where $G$ and $H$ have at most $k$ and $l$ rows respectively. On the other hand, from the theory of compact dual pairs ([Ho89], $[\mathrm{KV}]), \mathcal{P}\left(V_{n, k, l}\right)$ admits a decomposition

$$
\mathcal{P}\left(V_{n, k, l}\right) \cong \sum_{D, E} \rho_{n}^{D, E} \otimes W_{k, l}^{D, E}
$$


where $D$ and $E$ are Young diagrams with at most $k$ and $l$ rows respectively, $\rho_{n}^{D, E}$ is the irreducible representation of $\mathrm{GL}_{n}$ associated with $(D, E)$, and $W_{k, l}^{D, E}$ is a highest weight module for $\mathfrak{u}(k, l)_{\mathbb{C}} \cong \mathfrak{g l}_{k+l}(\mathbb{C})$. From the basis we have constructed, we obtain a basis for the space of all $\mathfrak{g l}_{k}(\mathbb{C}) \oplus \mathfrak{g l}_{l}(\mathbb{C})$ highest weight vectors in $W_{k, l}^{D, E}$.

This paper is arranged as follows. In Section [2 we set up some notation and review some results in HTW3 which we need in our construction. We construct bases for $\mathrm{TA}_{n, l_{1}, \ldots, l_{k}}$ and $\mathcal{P}\left(V_{n, k, l}\right)^{U_{n} \times U_{k} \times U_{l}}$ in Sections 3 and 4 respectively.

\section{Preliminaries}

In this section, we shall set up some notation and review some results in HTW3. which we need in our construction.

2.1. Young diagrams. A Young diagram $D$ is an array of square boxes arranged in left-justified horizontal rows, with each row no longer than the one above it ([Ful] $)$. We shall denote the number of rows in $D$ by $r(D)$. If $\lambda_{i}$ is the number of boxes in the row $i$ of $D$, then we shall write

$$
D=\left(\lambda_{1}, \ldots, \lambda_{r}\right)
$$

where $r=r(D)$. We also set

$$
|D|=\lambda_{1}+\lambda_{2}+\cdots+\lambda_{n} .
$$

If we flip $D$ over the diagonal line that slants down and to the right from the upper left-hand corner, we obtain its conjugate diagram $D^{t}$. For example, if $D=$ $(6,4,4,2)$, then $D^{t}=(4,4,3,3,1,1)$.

2.2. Representations of $\mathrm{GL}_{n}$. The irreducible finite-dimensional representations of $\mathrm{GL}_{n}$ are parametrized by ordered pairs $(D, E)$ of Young diagrams such that $r(D)+r(E) \leq n$. More precisely, let $B_{n}=A_{n} U_{n}$ be the standard Borel subgroup of upper triangular matrices in $\mathrm{GL}_{n}$, where $A_{n}$ is the diagonal torus in $\mathrm{GL}_{n}$ and $U_{n}$ is the maximal unipotent subgroup consisting of all $n \times n$ upper triangular matrices with 1's on the diagonal. For each $\lambda=\left(\lambda_{1}, \ldots, \lambda_{n}\right) \in \mathbb{Z}^{n}$, let $\psi_{n}^{\lambda}: A_{n} \rightarrow \mathbb{C}^{\times}$be the character given by

$$
\psi_{n}^{\lambda}\left[\operatorname{diag}\left(a_{1}, \ldots, a_{n}\right)\right]=a_{1}^{\lambda_{1}} a_{2}^{\lambda_{2}} \cdots a_{n}^{\lambda_{n}} .
$$

Let

$$
\mathcal{C}=\left\{\lambda=\left(\lambda_{1}, \ldots, \lambda_{n}\right) \in \mathbb{Z}^{n}: \lambda_{1} \geq \lambda_{2} \geq \cdots \geq \lambda_{n}\right\} .
$$

Then the semigroup $\widehat{A}_{n}^{+}$of dominant weights for $\mathrm{GL}_{n}$ is given by

$$
\widehat{A}_{n}^{+}=\left\{\psi_{n}^{\lambda}: \lambda \in \mathcal{C}\right\} .
$$

Note that each $\lambda \in \mathcal{C}$ can be written in the form

$$
\lambda=\left(d_{1}, d_{2}, \ldots, d_{r}, 0, \ldots, 0,-e_{s},-e_{s-1}, \ldots,-e_{1}\right),
$$

where $0 \leq r+s \leq n, d_{r}>0$ and $e_{s}>0$. Let $D$ and $E$ be the Young diagrams given by

$$
\begin{aligned}
& D=\left(d_{1}, d_{2}, \ldots, d_{r}\right), \\
& E=\left(e_{1}, e_{2}, \ldots, e_{s}\right) .
\end{aligned}
$$

Then the $\mathrm{GL}_{n}$ representation indexed by $(D, E)$ is the representation with highest weight $\psi_{n}^{\lambda}$. We shall write the character $\psi_{n}^{\lambda}$ as $\psi_{n}^{D, E}$ and shall denote the $\mathrm{GL}_{n}$ 
representation with highest weight $\psi_{n}^{D, E}$ by $\rho_{n}^{D, E}$. When $E=0$, we shall write $\psi_{n}^{D, 0}$ and $\rho_{n}^{D, 0}$ simply as $\psi_{n}^{D}$ and $\rho_{n}^{D}$ respectively.

2.3. Littlewood-Richardson tableaux and standard peeling. A LittlewoodRichardson tableau (LR tableau) $T$ is a skew diagram $F / D$ filled with positive integers 1 through $k$ for some $k$ which satisfies the following conditions:

(i) The numbers in a row are weakly increasing from left to right, and the numbers in a column are strictly increasing from top to bottom.

(ii) For every pair of positive integers $m$ and $p$, the number of times the number $m$ occurs in the first $p$ rows of $T$ is not larger than the number of times the number of times $m-1$ appears in the first $p-1$ rows of the tableau. The condition is interpreted as being vacuous when $m=1$.

If $E$ is a Young diagram, then by a banal tableau of shape $E$, we mean the tableau obtained by filling each column of $E$ from top to bottom with consecutive positive integers starting from 1.

In [HTW3, for a given LR tableau $T$, a banal tableau $B T$ and a "content preserving" map from $T$ to $B T$ are defined; i.e., each cell of $T$ is mapped to a cell in $B T$ with the same value. The map can be visualized as the process of successively removing the "vertical skew strips" from $T$ and reassembling them into columns of $B T$. This process is called "standard peeling". Thus $T$ is constructed by the reverse process of standard peeling. The contents of $B T$ are moved to the skew diagrams one column at a time, starting from the last column of $B T$. If $B T$ has shape $E$, then we shall say " $T$ is an LR tableau of shape $F / D$ and content $E$." Using standard peeling, we can associate $T$ with the matrix $M(T)=\left(m_{i j}\right)$ of nonnegative integers where $m_{i j}$ is the number of entries from the $j$-th column of $E$ that get put into the $i$-th column of $F / D$.

2.4. The leading monomial of $\delta_{T}(Y)$. In this subsection, we shall review some results in [HTW3] which we need in our construction. Fix positive integers $n, k$ and $l$ such that $k, l \leq n$. Let

$$
(X, Y)=\left(\begin{array}{cccccccccccc}
x_{11} & x_{12} & \cdot & \cdot & \cdot & x_{1 k} & y_{11} & y_{12} & \cdot & \cdot & \cdot & y_{1 l} \\
x_{21} & x_{22} & \cdot & \cdot & \cdot & x_{2 k} & y_{21} & y_{22} & \cdot & \cdot & \cdot & y_{2 l} \\
\cdot & \cdot & & & \cdot & \cdot & \cdot & & & \cdot \\
\cdot & \cdot & & & \cdot & \cdot & \cdot & & \\
\cdot & \cdot & & & & \cdot & \cdot & \cdot & & & \cdot \\
x_{n 1} & x_{n 2} & \cdot & \cdot & \cdot & x_{n k} & y_{n 1} & y_{n 2} & \cdot & \cdot & \cdot & y_{n l}
\end{array}\right)
$$

be the system of standard coordinates on the space $\mathrm{M}_{n, k+l}(\mathbb{C})$ of $n \times(k+l)$ complex matrices. Let $D$ and $E$ be Young diagrams with at most $k$ and $l$ rows respectively and $F$ a Young diagram such that $\rho_{n}^{D} \otimes \rho_{n}^{E}$ contains $\rho_{n}^{F}$, or equivalently the Littlewood-Richardson coefficient $([\mathrm{Ful}]) c_{D, E}^{F} \neq 0$. Let $T$ be a LittlewoodRichardson tableau of shape $F / D$ and content $E$. Then HTW3 describes a $\mathrm{GL}_{n} \times \mathrm{GL}_{k} \times \mathrm{GL}_{l}$ highest weight vector $\Delta_{T}=\Delta_{(D, E, F), T}$ in $\mathcal{P}\left(\mathrm{M}_{n, k+l}(\mathbb{C})\right)$ with weight $\psi_{n}^{F} \times \psi_{k}^{D} \times \psi_{l}^{E}$ and computes its leading monomial with respect to a certain monomial ordering in $\mathcal{P}\left(\mathrm{M}_{n, k+l}(\mathbb{C})\right)$. Let

$$
\begin{aligned}
D^{t} & =\left(d_{1}, d_{2}, \ldots, d_{r}\right), \\
E^{t} & =\left(e_{1}, e_{2}, \ldots, e_{s}\right), \\
F^{t} & =\left(f_{1}, f_{2}, \ldots, f_{t}\right),
\end{aligned}
$$


where $D^{t}, E^{t}$ and $F^{t}$ are the conjugate diagrams of $D, E$ and $F$ respectively. This means that the entries in equation (11) represent the lengths of the columns in the diagrams $D, E$ and $F$. Then by the choice of the monomial ordering, the leading monomial of $\Delta_{T}$ contains the factor

$$
x_{D}=\prod_{j=1}^{r}\left(\prod_{i=1}^{d_{j}} x_{i i}\right) .
$$

Thus they consider the polynomial $\delta_{T}(Y)=\delta_{(D, E, F), T}(Y)$, which is the sum of all terms in $\Delta_{T}$ containing the factor $x_{D}$, and compute its leading monomial. The polynomial $\delta_{T}(Y)$ is important to our construction, so we shall review its definition and properties in this subsection.

Let

$$
B=\left(\begin{array}{cccc}
\beta_{11} & \beta_{12} & \cdots & \beta_{1 s} \\
\beta_{21} & \beta_{22} & \cdots & \beta_{2 s} \\
\cdot & & & \cdot \\
\cdot & & & \cdot \\
\cdot & & & \cdot \\
\beta_{t 1} & \beta_{t 2} & \cdots & \beta_{t s}
\end{array}\right)
$$

be a $t \times r$ matrix of indeterminates. Let $\widetilde{Y}_{0}$ be the $|E| \times|E|$ matrix defined as follows: $\widetilde{Y}_{0}$ is divided into st blocks, and its $(i, j)$-th block is given by

$$
\left(\widetilde{Y}_{0}\right)_{i j}=\beta_{i j}\left(\begin{array}{cccccc}
y_{d_{i}+1,1} & y_{d_{i}+1,2} & \cdot & \cdot & \cdot & y_{d_{i}+1, e_{j}} \\
y_{d_{i}+2,1} & y_{d_{i}+2,2} & \cdot & \cdot & \cdot & y_{d_{i}+2, e_{j}} \\
\cdot & & & & \cdot \\
\cdot & & & & \cdot \\
\cdot & & & & & \cdot \\
y_{f_{i}, 1} & y_{f_{i}, 2} & \cdot & \cdot & \cdot & y_{f_{i}, e_{j}}
\end{array}\right) .
$$

The determinant of $\widetilde{Y}_{0}$ is a polynomial in $Y=\left(y_{i j}\right)$ and $\beta=\left(\beta_{a b}\right)$, so that it can be expressed as

$$
\operatorname{det} \tilde{Y}_{0}=\sum_{M} p_{M}(Y) \beta^{M},
$$

where $M=\left(m_{i j}\right)$ is a matrix of nonnegative integers, $\beta^{M}=\prod_{i, j} \beta_{i j}^{m_{i j}}$, and $p_{M}(Y)$ is a polynomial in the variables $\left\{y_{i j}\right\}$. Recall that $T$ is an LR tableau of shape $F / D$ and content $E$. Let

$$
M(T)=\left(m_{i h}\right),
$$

where $m_{i h}$ is the number of elements from the $h$-th column of $E$ which get put into the $i$-th column of $F / D$. Set

$$
\delta_{T}(Y)=p_{M(T)}(Y)
$$

We now introduce the following monomial ordering on $\mathcal{P}\left(\mathrm{M}_{n, l}(\mathbb{C})\right)=\mathbb{C}[Y]$ :

(a) Set $y_{i j}>y_{a b}$ if and only if either $j<b$ or $j=b$ and $i<a$. Thus

$$
y_{11}>y_{21}>\cdots>y_{n 1}>y_{12}>y_{22}>\cdots>y_{n l} \text {. }
$$


(b) Extend the ordering in (a) to an ordering between all monomials in the $y_{i j}$ 's by the graded lexicographic order (see CLO ). That is, if $y^{M_{1}}$ and $y^{M_{2}}$ are two monomials and the total degree of $y^{M_{1}}$ is higher than that of $y^{M_{2}}$, then $y^{M_{1}}>y^{M_{2}}$. If $y^{M_{1}}$ and $y^{M_{2}}$ have the same total degree, then we shall write them as

$$
\begin{aligned}
& y^{M_{1}}=y_{i_{1}, j_{1}} y_{i_{2}, j_{2}} \cdots, \\
& y^{M_{2}}=y_{a_{1}, b_{1}} y_{a_{2}, b_{2}} \cdots,
\end{aligned}
$$

where the factors of each monomial are written in decreasing order. If $k$ is the first index such that $y_{i_{k}, j_{k}} \neq y_{a_{k}, b_{k}}$, then $y^{M_{1}}>y^{M_{2}}$ if and only if $y_{i_{k}, j_{k}}>y_{a_{k}, b_{k}}$.

Proposition 2.4.1. ([HTW3] ) Let $e_{T}(Y)$ be the leading monomial of $\delta_{T}(Y)$ with respect to the above monomial ordering. Then

$$
e_{T}(Y)=\prod_{\mathbf{b} \in T} y_{a(\mathbf{b}) c(\mathbf{b})},
$$

where $a(\mathbf{b})$ is the row of $F$ in which the box $\mathbf{b}$ lies, and $c(\mathbf{b})$ is the entry in $\mathbf{b}$.

Remarks. Let $p$ and $q$ be the numbers of rows in the diagrams $F$ and $E$ respectively, that is, $p=r(F)$ and $q=r(E)$. We note that $\delta_{T}(Y)$ in fact only depends on the variables in the $p \times q$ submatrix

$$
\left(\begin{array}{cccccc}
y_{11} & y_{12} & \cdot & \cdot & \cdot & y_{1 q} \\
y_{21} & y_{22} & \cdot & \cdot & \cdot & y_{2 q} \\
\cdot & \cdot & & & \cdot \\
\cdot & \cdot & & & & \cdot \\
\cdot & \cdot & & & & \cdot \\
y_{p 1} & y_{p 2} & \cdot & \cdot & \cdot & y_{p q}
\end{array}\right)
$$

of $Y$. For any $s \geq p$ and $t \geq q$, let $\pi: \mathrm{M}_{s, t}(\mathbb{C}) \rightarrow \mathrm{M}_{p, q}(\mathbb{C})$ be the linear projection

$$
Z=\left(\begin{array}{cccccc}
z_{11} & z_{12} & \cdot & \cdot & \cdot & z_{1 t} \\
z_{21} & z_{22} & \cdot & \cdot & \cdot & z_{2 t} \\
\cdot & \cdot & & & \cdot \\
\cdot & \cdot & & & \cdot \\
\cdot & \cdot & & & \cdot \\
z_{s 1} & z_{s 2} & \cdot & \cdot & \cdot & z_{s t}
\end{array}\right) \rightarrow\left(\begin{array}{cccccc}
z_{11} & z_{12} & \cdot & \cdot & \cdot & z_{1 q} \\
z_{21} & z_{22} & \cdot & \cdot & \cdot & z_{2 q} \\
\cdot & \cdot & & & \cdot \\
\cdot & \cdot & & & \cdot \\
\cdot & \cdot & & & \cdot \\
z_{p 1} & z_{p 2} & \cdot & \cdot & \cdot & z_{p q}
\end{array}\right) .
$$

Using $\pi, \delta_{T}$ can be identified with the following polynomial on $\mathrm{M}_{s, t}(\mathbb{C})$ :

$$
Z \rightarrow \delta_{T}[\pi(Z)] \text {. }
$$

We shall make this identification in Section 4 when we compute the leading monomials of some highest weight vectors in $\mathcal{P}\left(V_{n, k, l}\right)$.

\section{A BASIS FOR THE $k$-FOLD TENSOR PRODUCT ALGEBRA FOR GL G $_{n}$}

3.1. The algebra $\mathcal{P}\left(V_{n, l_{1}, \ldots, l_{k}}\right)$. The construction in HTW3 can be generalized to an algebra which describes $k$-fold tensor products of irreducible $\mathrm{GL}_{n}$ modules. Specifically, let $l_{1}, \ldots, l_{k}$ be positive integers less than or equal to $n$ and consider the space

$$
V_{n, l_{1}, \ldots, l_{k}}=\bigoplus_{j=1}^{k} \mathrm{M}_{n, l_{j}}(\mathbb{C}) .
$$


Here $\mathrm{M}_{s, t}(\mathbb{C})$ denotes the space of all $s \times t$ complex matrices. Let $\mathrm{GL}_{n} \times \mathrm{GL}_{l_{1}} \times$ $\cdots \times \mathrm{GL}_{l_{k}}$ act on $V_{n, l_{1}, l_{2}, \ldots, l_{k}}$ by

$$
\left[\left(g, h_{1}, \ldots, h_{k}\right),\left(X_{1}, \ldots, X_{k}\right)\right] \rightarrow\left(\left(g^{t}\right)^{-1} X_{1} h_{1}^{-1},\left(g^{t}\right)^{-1} X_{2} h_{2}^{-1}, \ldots,\left(g^{t}\right)^{-1} X_{k} h_{k}^{-1}\right),
$$

where $g \in \mathrm{GL}_{n}, h_{j} \in \mathrm{GL}_{l_{j}}$ and $X_{j} \in \mathrm{M}_{n, l_{j}}(\mathbb{C})$ for $1 \leq j \leq k$. This action induces an action of $\mathrm{GL}_{n} \times \mathrm{GL}_{l_{1}} \times \cdots \times \mathrm{GL}_{l_{k}}$ on $\mathcal{P}\left(V_{n, l_{1}, \ldots, l_{k}}\right)$. In this section, we will construct a basis for the algebra

$$
\mathrm{TA}_{n, l_{1}, \ldots, l_{k}}=\mathcal{P}\left(V_{n, l_{1}, \ldots, l_{k}}\right)^{U_{n} \times U_{l_{1}} \times \cdots \times U_{l_{k}}}
$$

of $\mathrm{GL}_{n} \times \mathrm{GL}_{l_{1}} \times \cdots \times \mathrm{GL}_{l_{k}}$ highest weight vectors in $\mathcal{P}\left(V_{n, l_{1}, \ldots, l_{k}}\right)$.

Recall that $l_{j} \leq n$ for $n=1, \ldots, k$. We first consider the $\mathrm{GL}_{n} \times \mathrm{GL}_{l_{1}} \times \cdots \times \mathrm{GL}_{l_{k}}$ module structure of $\mathcal{P}\left(V_{n, l_{1}, \ldots, l_{k}}\right)$. By the $\left(\mathrm{GL}_{n}, \mathrm{GL}_{l_{j}}(\mathbb{C})\right)$-duality ([Ho95]), we have

$$
\begin{aligned}
\mathcal{P}\left(V_{n, l_{1}, \ldots, l_{k}}\right) & =\mathcal{P}\left(\mathrm{M}_{n, l_{1}}(\mathbb{C}) \oplus \cdots \oplus \mathrm{M}_{n, l_{k}}(\mathbb{C})\right) \\
& \cong \mathcal{P}\left(\mathrm{M}_{n, l_{1}}(\mathbb{C})\right) \otimes \cdots \otimes \mathcal{P}\left(\mathrm{M}_{n, l_{k}}(\mathbb{C})\right) \\
& \cong \sum_{r\left(D_{1}\right) \leq l_{1}}\left(\rho_{n}^{D_{1}} \otimes \rho_{l_{1}}^{D_{1}}\right) \otimes \cdots \otimes \sum_{r\left(D_{k}\right) \leq l_{k}}\left(\rho_{n}^{D_{k}} \otimes \rho_{l_{k}}^{D_{k}}\right) \\
& \cong \sum_{\substack{r\left(D_{j}\right) \leq l_{j} \\
1 \leq j \leq k}}\left(\rho_{n}^{D_{1}} \otimes \cdots \otimes \rho_{n}^{D_{k}}\right) \otimes \rho_{l_{1}}^{D_{1}} \otimes \cdots \otimes \rho_{l_{k}}^{D_{k}} .
\end{aligned}
$$

Taking $U_{l_{1}} \times \cdots \times U_{l_{k}}$ invariants gives

$$
\mathcal{P}\left(V_{n, l_{1}, \ldots, l_{k}}\right)^{U_{l_{1}} \times \cdots \times U_{l_{k}}}=\sum_{\substack{r\left(D_{j}\right) \leq l_{j} \\ 1 \leq j \leq k}}\left(\rho_{n}^{D_{1}} \otimes \cdots \otimes \rho_{n}^{D_{k}}\right) \otimes\left(\rho_{l_{1}}^{D_{1}}\right)^{U_{l_{1}}} \otimes \cdots \otimes\left(\rho_{l_{k}}^{D_{k}}\right)^{U_{l_{k}}} .
$$

By the theory of highest weight,

$$
\operatorname{dim}\left[\left(\rho_{l_{1}}^{D_{1}}\right)^{U_{l_{1}}} \otimes \cdots \otimes\left(\rho_{l_{k}}^{D_{k}}\right)^{U_{l_{k}}}\right]=1
$$

Thus the algebra $\mathcal{P}\left(V_{n, l_{1}, \ldots, l_{k}}\right)^{U_{l_{1}} \times \cdots \times U_{l_{k}}}$ contains one copy of the $k$-fold tensor product $\rho_{n}^{D_{1}} \otimes \cdots \otimes \rho_{n}^{D_{k}}$ for each $k$-tuple $\left(D_{1}, \ldots, D_{k}\right)$ of Young diagrams with $r\left(D_{j}\right) \leq l_{j}$ for $1 \leq j \leq k$, and the algebra

$$
\mathcal{P}\left(V_{n, l_{1}, \ldots, l_{k}}\right)^{U_{n} \times U_{l_{1}} \times \cdots \times U_{l_{k}}}=\left[\mathcal{P}\left(V_{n, l_{1}, \ldots, l_{k}}\right)^{U_{l_{1}} \times \cdots \times U_{l_{k}}}\right]^{U_{n}}
$$

of $\mathrm{GL}_{n}$ highest weight vectors in $\mathcal{P}\left(V_{n, l_{1}, \ldots, l_{k}}\right)^{U_{l_{1}} \times \cdots \times U_{l_{k}}}$ describes the decomposition of $\rho_{n}^{D_{1}} \otimes \cdots \otimes \rho_{n}^{D_{k}}$ into a sum of irreducible $\mathrm{GL}_{n}$ representations. Thus we shall call this algebra a $k$-fold tensor product algebra for $\mathrm{GL}_{n}$.

The algebra $\mathcal{P}\left(V_{n, l_{1}, \ldots, l_{k}}\right)^{U_{n} \times U_{l_{1}} \times \cdots \times U_{l_{k}}}$ also contains another kind of branching information. Let $l=\sum_{j=1}^{k} l_{j}$. Then

$$
\mathcal{P}\left(V_{n, l_{1}, \ldots, l_{k}}\right) \cong \mathcal{P}\left(\mathrm{M}_{n, l}(\mathbb{C})\right),
$$

which carries a natural $\mathrm{GL}_{n} \times \mathrm{GL}_{l}$ action. By the $\left(\mathrm{GL}_{n}, \mathrm{GL}_{l}\right)$-duality,

$$
\mathcal{P}\left(\mathrm{M}_{n, l}(\mathbb{C})\right) \cong \sum_{r(D) \leq \min (n, l)} \rho_{n}^{D} \otimes \rho_{l}^{D} .
$$

If we take $U_{n}$ invariants, we obtain

$$
\mathcal{P}\left(\mathrm{M}_{n, l}(\mathbb{C})\right)^{U_{n}} \cong \sum_{r(D) \leq \min (n, l)}\left(\rho_{n}^{D}\right)^{U_{n}} \otimes \rho_{l}^{D},
$$


which contains one copy of $\rho_{l}^{D}$ for each $D$ with $r(D) \leq \min (n, l)$. Thus the algebra

$$
\mathcal{P}\left(V_{n, l_{1}, \ldots, l_{k}}\right)^{U_{n} \times U_{l_{1}} \times \cdots \times U_{l_{k}}}=\left[\mathcal{P}\left(\mathrm{M}_{n, l}(\mathbb{C})\right)^{U_{n}}\right]^{U_{l_{1}} \times \cdots \times U_{l_{k}}}
$$

describes the decomposition of an irreducible $\mathrm{GL}_{l}$ representation into a sum of irreducible $\mathrm{GL}_{l_{1}}(\mathbb{C}) \times \cdots \times \mathrm{GL}_{l_{k}}(\mathbb{C})$ representations. Since $\mathcal{P}\left(V_{n, l_{1}, \ldots, l_{k}}\right)^{U_{n} \times U_{l_{1}} \times \cdots \times U_{l_{k}}}$ carries two kinds of branching information associated with the see-saw dual pairs

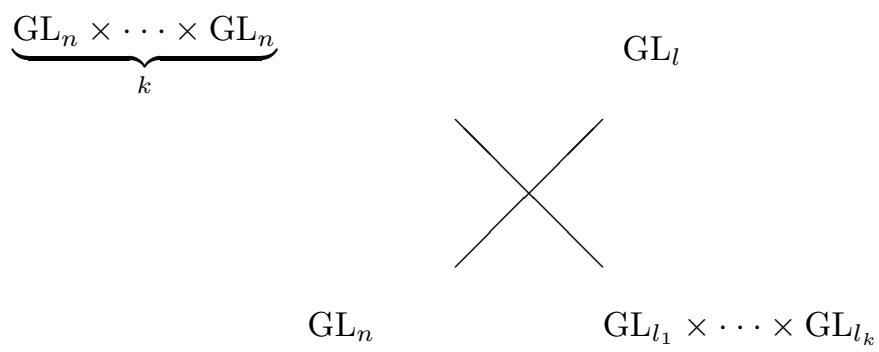

it is a reciprocity algebra in the sense of [HTW1].

3.2. Highest weight vectors in $\mathcal{P}\left(V_{n, l_{1}, \ldots, l_{k}}\right)$. Write the standard coordinates on $\mathrm{M}_{n, l_{j}}(\mathbb{C})$ as

$$
X^{(j)}=\left(\begin{array}{cccccc}
x_{11}^{(j)} & x_{12}^{(j)} & \cdot & \cdot & \cdot & x_{1, l_{j}}^{(j)} \\
x_{21}^{(j)} & x_{22}^{(j)} & \cdot & \cdot & \cdot & x_{2, l_{j}}^{(j)} \\
\cdot & \cdot & & & \cdot \\
\cdot & \cdot & & & \cdot \\
\cdot & \cdot & & & \cdot \\
x_{n 1}^{(j)} & x_{n 2}^{(j)} & \cdot & \cdot & \cdot & x_{n, l_{j}}^{(j)}
\end{array}\right) .
$$

and write $X \in V_{n, l_{1}, \ldots, l_{k}}$ as

$$
X=\left(X^{(1)}, X^{(2)}, \ldots, X^{(k)}\right) .
$$

Let $D_{1}, \ldots, D_{k}$ be Young diagrams such that $r\left(D_{j}\right) \leq l_{j}$ for all $j$. We consider the $k$-fold tensor product

$$
\rho_{n}^{D_{1}} \otimes \rho_{n}^{D_{2}} \otimes \cdots \otimes \rho_{n}^{D_{k}} .
$$

For Young diagrams $D, E$ and $F$, we denote by $c_{D, E}^{F}$ the number of LR tableaux of shape $F / D$ and content $E$. By applying the Littlewood-Richardson rule repeatedly, we find that

$$
\begin{aligned}
\rho_{n}^{D_{1}} \otimes \rho_{n}^{D_{2}} \otimes \cdots \otimes \rho_{n}^{D_{k}} & =\sum_{F_{1}, F_{2}, \ldots, F_{k-1}}\left(c_{D_{1}, D_{2}}^{F_{1}} c_{F_{1}, D_{3}}^{F_{2}} \cdots c_{F_{k-2}, D_{k}}^{F_{k-1}}\right) \rho_{n}^{F_{k-1}} \\
& =\sum_{F}\left(\sum_{F_{1}, F_{2}, \ldots, F_{k-2}} c_{D_{1}, D_{2}}^{F_{1}} c_{F_{1}, D_{3}}^{F_{2}} \cdots c_{F_{k-2}, D_{k}}^{F}\right) \rho_{n}^{F},
\end{aligned}
$$

where $F=F_{k-1}$. Thus the multiplicity of $\rho_{n}^{F}$ in $\rho_{n}^{D_{1}} \otimes \rho_{n}^{D_{2}} \otimes \cdots \otimes \rho_{n}^{D_{k}}$ is given by the sum

$$
\sum_{F_{1}, F_{2}, \ldots, F_{k-2}} c_{D_{1}, D_{2}}^{F_{1}} c_{F_{1}, D_{3}}^{F_{2}} \cdots c_{F_{k-2}, D_{k}}^{F}
$$

which is equal to the number of all $(k-1)$-tuples $\left(T_{1}, \ldots, T_{k-1}\right)$ of LittlewoodRichardson tableaux with the following properties: there exist Young diagrams 
$F_{1}, \ldots, F_{k-2}$ such that for each $1 \leq j \leq k-1, T_{j}$ is of shape $F_{j} / F_{j-1}$ and content $D_{j+1}$, where $F_{0}=D_{1}$. Let

$$
\begin{gathered}
D_{j}^{t}=\left(d_{1}^{(j)}, \ldots, d_{r_{j}}^{(j)}\right) \quad(1 \leq j \leq k), \\
F_{m}^{t}=\left(f_{1}^{(m)}, \ldots, f_{t_{m}}^{(m)}\right) \quad(0 \leq m \leq k-2),
\end{gathered}
$$

and

$$
F^{t}=\left(f_{1}, \ldots, f_{t}\right)
$$

For each $1 \leq j \leq k$, write

$$
\begin{gathered}
X_{a b}^{(j)}=\left(\begin{array}{cccccc}
x_{11}^{(j)} & x_{12}^{(j)} & \cdot & \cdot & \cdot & x_{1 b}^{(j)} \\
x_{21}^{(j)} & x_{22}^{(j)} & \cdot & \cdot & \cdot & x_{2 b}^{(j)} \\
\cdot & & & & \cdot \\
\cdot & & & & \cdot \\
\cdot & & & & \cdot \\
x_{a 1}^{(j)} & x_{a 2}^{(j)} & \cdot & \cdot & \cdot & x_{a b}^{(j)}
\end{array}\right), \\
\widetilde{X}_{i h}^{(1)}=X_{f_{i}, d_{h}^{(1)}}^{(1)},
\end{gathered}
$$

and for $m \geq 2$,

$$
\widetilde{X}_{i h}^{(m)}=\beta_{i h}^{(m)} X_{f_{i}, d_{h}^{(m)}}^{(m)}
$$

Here

$$
B^{(j)}=\left(\begin{array}{cccc}
\beta_{11}^{(j)} & \beta_{12}^{(j)} & \cdots & \beta_{1, r_{j}}^{(j)} \\
\beta_{21}^{(j)} & \beta_{22}^{(j)} & \cdots & \beta_{2, r_{j}}^{(j)} \\
\cdot & & & \cdot \\
\cdot & & & \cdot \\
\cdot & & & \cdot \\
\beta_{t 1}^{(j)} & \beta_{t 2}^{(j)} & \cdots & \beta_{t, r_{j}}^{(j)}
\end{array}\right)
$$

is a $t \times r_{j}$ matrix of indeterminates. Let

$$
\widetilde{X}=\left(\widetilde{X}^{(1)}, \widetilde{X}^{(2)}, \ldots, \widetilde{X}^{(k)}\right)
$$

where

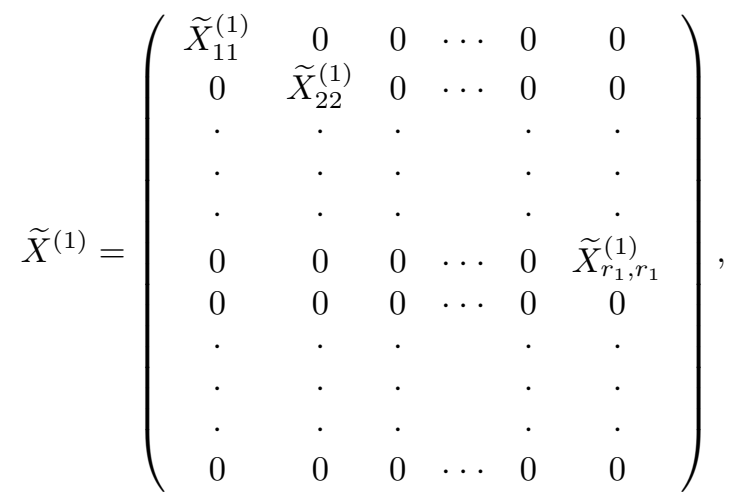


and for $j \geq 2$,

$$
\widetilde{X}^{(j)}=\left(\begin{array}{cccc}
\widetilde{X}_{11}^{(j)} & \widetilde{X}_{12}^{(j)} & \ldots & \widetilde{X}_{1, r_{j}}^{(j)} \\
\widetilde{X}_{21}^{(j)} & \widetilde{X}_{22}^{(j)} & \ldots & \widetilde{X}_{2, r_{j}}^{(j)} \\
\cdot & \cdot & & \cdot \\
\cdot & \cdot & & \cdot \\
\cdot & \cdot & & \cdot \\
\widetilde{X}_{t 1}^{(j)} & \widetilde{X}_{t 2}^{(j)} & \ldots & \widetilde{X}_{t, r_{j}}^{(j)}
\end{array}\right) .
$$

We now consider the determinant $\operatorname{det} \widetilde{X}$ of $\tilde{X}$. If we expand this determinant as a polynomial in the $\beta_{i h}^{(j)}$,s, we obtain

$$
\operatorname{det} \tilde{X}=\sum_{M} \tilde{\Delta}_{\left(D_{1}, \ldots, D_{k}, F\right), M} \beta^{M},
$$

where $M=\left(M_{2}, \ldots, M_{k}\right)$,

$$
\beta^{M}=\prod_{j=2}^{k}\left[\beta^{(j)}\right]^{M_{j}},
$$

and for each $2 \leq j \leq k, M_{j}=\left(m_{i h}^{(j)}\right)$ is a $t \times r_{j}$ matrix of nonnegative integers and

$$
\left[\beta^{(j)}\right]^{M_{j}}=\prod_{i, h}\left(\beta_{i h}^{(j)}\right)^{m_{i h}^{(j)}} .
$$

It can be shown that $\operatorname{det} \widetilde{X}$ as well as the coefficient $\tilde{\Delta}_{\left(D_{1}, \ldots, D_{k}, F\right), M}$ of $\beta^{M}$ in equation (9) are $\mathrm{GL}_{n} \times \mathrm{GL}_{l_{1}} \times \mathrm{GL}_{l_{2}} \times \cdots \times \mathrm{GL}_{l_{k}}$ highest weight vectors of weight

$$
\psi_{n}^{F} \times \psi_{l_{1}}^{D_{1}} \times \cdots \times \psi_{l_{k}}^{D_{k}} .
$$

We omit its proof as it is similar to the case $k=2$, which is treated in [HTW3.

We now let $F_{0}=D_{1}, F_{2}, \ldots, F_{k-1}=F$ be Young diagrams such that $r\left(D_{j}\right) \leq l_{j}$ for $1 \leq j \leq k$, and let $T_{1}, \ldots, T_{k-1}$ be Littlewood-Richardson tableaux such that for each $1 \leq j \leq k-1, T_{j}$ is of shape $F_{j} / F_{j-1}$ and content $D_{j+1}$. Let

$$
M\left(T_{1}, \ldots, T_{k-1}\right)=\left(M_{2}, \ldots, M_{k}\right),
$$

where for each $2 \leq j \leq k, M_{j}=M_{j}\left(T_{j-1}\right)=\left(m_{i h}^{(j)}\right)$ is a $t \times r_{j}$ matrix, and $m_{i h}^{(j)}$ is the number of elements from the $h$-th column of $D_{j}$ which get put into the $i$-th column of $F_{j} / F_{j-1}$. We consider the coefficient of $\beta^{M\left(T_{1}, \ldots, T_{k-1}\right)}$ in $\operatorname{det} \tilde{X}$,

$$
\tilde{\Delta}_{\left(D_{1}, \ldots, D_{k}, F\right), M\left(T_{1}, \ldots, T_{k-1}\right)} .
$$

It is a $\mathrm{GL}_{n} \times \mathrm{GL}_{l_{1}} \times \mathrm{GL}_{l_{2}} \times \cdots \times \mathrm{GL}_{l_{k}}$ highest weight vector of weight $\psi_{n}^{F} \times \psi_{l_{1}}^{D_{1}} \times$ $\cdots \times \psi_{l_{k}}^{D_{k}}$.

3.3. Leading monomial of $\tilde{\Delta}_{\left(D_{1}, \ldots, D_{k}, F\right), M\left(T_{1}, \ldots, T_{k-1}\right)}$. We now define a monomial ordering on $\mathcal{P}\left(V_{n, l_{1}, \ldots, l_{k}}\right)$ as follows. We shall denote a general monomial in $\mathcal{P}\left(V_{n, l_{1}, \ldots, l_{k}}\right)$ by

$$
x^{A}=\prod_{j, i, h}\left(x_{i h}^{(j)}\right)^{a_{i h}^{(j)}},
$$

where $A=\left(A_{1}, \ldots, A_{k}\right), A_{j}=\left(a_{i h}^{(j)}\right)$ for each $1 \leq j \leq k$. 
(Ord 1) If $i<j$, then

$$
x_{a b}^{(i)}>x_{c d}^{(j)}
$$

for all $1 \leq a, c \leq n, 1 \leq b \leq l_{i}$ and $1 \leq d \leq l_{j}$.

(Ord 2) For each $1 \leq j \leq k$,

$$
x_{a b}^{(j)}>x_{c d}^{(j)}
$$

if and only if $b<d$, or $b=d$ and $a<c$.

By (Ord 1) and (Ord 2), we have

$$
x_{11}^{(1)}>x_{21}^{(1)}>\cdots>x_{n 1}^{(1)}>x_{12}^{(1)}>x_{22}^{(1)}>\cdots>x_{n, l_{1}}^{(1)}>x_{11}^{(2)}>x_{21}^{(2)}>\cdots>x_{n, l_{k}}^{(k)} .
$$

We now extend the ordering in (Ord 1) and (Ord 2) to an ordering between all monomials on the $x_{i h}^{(j)}$ 's by the graded lexicographic order (see [CLO]). The specific conditions are given in (Ord 3)-(Ord 4) below:

(Ord 3) If the total degree of $x^{A}$ is higher than that of $x^{L}$, then

$$
x^{A}>x^{L} .
$$

(Ord 4) If $x^{A}$ and $x^{L}$ have the same total degree, we shall arrange the variables which occur in $x^{A}$ and $x^{L}$ in decreasing order. Then $x^{A}>x^{L}$ if and only if $x^{A}$ contains the higher first nonequal variable.

Lemma 3.3.1. The leading monomial $\operatorname{LM}(\tilde{\Delta})$ of $\tilde{\Delta}=\tilde{\Delta}_{\left(D_{1}, \ldots, D_{k}, F\right), M\left(T_{1}, \ldots, T_{k-1}\right)}$ is given by

$$
\operatorname{LM}(\tilde{\Delta})=\left[\prod_{m=1}^{r_{1}}\left(\prod_{i=1}^{d_{m}^{(1)}} x_{i i}^{(1)}\right)\right]\left[\prod_{j=1}^{k-1}\left(\prod_{\mathbf{b} \in T_{j}} x_{a_{j}(\mathbf{b}) c_{j}(\mathbf{b})}^{(j+1)}\right)\right],
$$

where for $1 \leq j \leq k-1$ and $\mathbf{b} \in T_{j}, a_{j}(\mathbf{b})$ is the row of $F_{j}$ in which the box $\mathbf{b}$ lies, and $c_{j}(\mathbf{b})$ is the entry in $\mathbf{b}$.

Proof. First we note that the monomials of $\tilde{\Delta}$ are of the form

$$
x^{A}=\left[x^{(1)}\right]^{A_{1}}\left[x^{(2)}\right]^{A_{2}} \cdots\left[x^{(k)}\right]^{A_{k}},
$$

where $A=\left(A_{1}, A_{2}, \ldots, A_{k}\right)$, and for each $j, A_{j}=\left(a_{s t}^{(j)}\right)$ is an $n \times l_{j}$ matrix of nonnegative integers, and

$$
\left[x^{(j)}\right]^{A_{j}}=\prod_{s, t}\left(x_{s t}^{(j)}\right)^{a_{s t}^{(j)}} .
$$

We shall call $\left[x^{(j)}\right]^{A_{j}}$ the $x^{(j)}$-part of $x^{A}$. In addition, since $\tilde{\Delta}$ is the coefficient of $\beta^{M\left(T_{1}, \ldots, T_{k-1}\right)}$ in the expansion of $\operatorname{det} \widetilde{X}, d_{p}^{(1)}$ and $m_{i h}^{(j)}$ of the variables occurring in $x^{A}$ are chosen from the submatrices $\widetilde{X}_{p p}^{(1)}$ and $\widetilde{X}_{i h}^{(j)}$, respectively.

For each $1 \leq j \leq k$, let $L_{j}\left(X^{(j)}\right)$ be the $x^{(j)}$-part of $\operatorname{LM}(\tilde{\Delta})$. For each $1 \leq j \leq$ $k-1$, let $\delta_{T_{1}, \ldots, T_{k-1}}^{(j)}$ be the coefficient of

$$
\prod_{m=1}^{j} L_{m}\left(X^{(m)}\right)
$$


in $\tilde{\Delta}$. In other words,

$$
\delta_{T_{1}, \ldots, T_{k-1}}^{(j)} \prod_{m=1}^{j} L_{m}\left(X^{(m)}\right)
$$

is equal to the sum of all the terms in $\tilde{\Delta}$ that contain

$$
\prod_{m=1}^{j} L_{m}\left(X^{(m)}\right)
$$

as a factor. By the definition of the monomial ordering (Ord 1)-(Ord 4), $L_{j+1}\left(X^{(j+1)}\right)$ is the largest $x^{(j+1)}$-part which appears in the monomials occurring in $\delta_{T_{1}, \ldots, T_{k-1}}^{(j)}$. Thus to compute $L_{j+1}\left(X^{(j+1)}\right)$, we need to have a more precise description of $\delta_{T_{1}, \ldots, T_{k-1}}^{(j)}$.

For each $1 \leq j \leq k-1$, let $\tilde{X}[j]$ be the submatrix of $\tilde{X}$ defined as follows:

$$
\widetilde{X}[j]=\left(\widetilde{X}^{(j+1)}[j], \widetilde{X}^{(j+2)}[j], \ldots, \widetilde{X}^{(k)}[j]\right),
$$

where for each $j+1 \leq p \leq k, 1 \leq a \leq t, 1 \leq b \leq r_{p}$,

$$
\widetilde{X}^{(p)}[j]=\left(\begin{array}{cccc}
\widetilde{X}_{11}^{(p)}[j] & \widetilde{X}_{12}^{(p)}[j] & \cdots & \widetilde{X}_{1, r_{p}}^{(p)}[j] \\
\widetilde{X}_{21}^{(p)}[j] & \widetilde{X}_{22}^{(p)}[j] & \cdots & \widetilde{X}_{2, r_{p}}^{(p)}[j] \\
\cdot & \cdot & & \cdot \\
\cdot & \cdot & & \cdot \\
\cdot & \cdot & & \cdot \\
\widetilde{X}_{t 1}^{(p)}[j] & \widetilde{X}_{t 2}^{(p)}[j] & \cdots & \widetilde{X}_{t, r_{p}}^{(p)}[j]
\end{array}\right),
$$

$$
\tilde{X}_{a b}^{(p)}[j]= \begin{cases}\text { the matrix obtained by eliminating } & \\ \text { the top } f_{a}^{(j-1)} \text { rows from } \widetilde{X}_{a b}^{(p)}, & \text { if } m_{a b}^{(p)} \neq 0, \\ \text { the }\left(f_{a}-f_{a}^{(j-1)}\right) \times d_{b}^{(p)} \text { zero matrix, } & \text { if } m_{a b}^{(p)}=0 .\end{cases}
$$

For $1 \leq j \leq k-1$, let

$$
e^{(j+1)}\left(T_{j}\right)=\prod_{\mathbf{b} \in T_{j}} x_{a_{j}(\mathbf{b}) c_{j}(\mathbf{b})}^{(j+1)}
$$

We claim that

$$
L_{j}\left(X^{(j)}\right)= \begin{cases}\prod_{j=1}^{r_{1}}\left(\prod_{i=1}^{d_{j}} x_{i i}\right), & j=1, \\ e^{(j)}\left(T_{j-1}\right), & 2 \leq j \leq k,\end{cases}
$$

and for $1 \leq j \leq k-1$,

$$
\begin{aligned}
\delta_{T_{1}, \ldots, T_{k-1}}^{(j)}= & \text { the coefficient of } \prod_{p=j+1}^{k}\left(\beta^{(p)}\right)^{M_{p}} \text { in the expansion } \\
& \text { of the determinant } \operatorname{det} \widetilde{X}[j] .
\end{aligned}
$$

(Recall that for each $2 \leq p \leq k, M_{p}=M_{p}\left(T_{p-1}\right)=\left(m_{i h}^{(p)}\right)$ is a $t \times r_{p}$ matrix, and $m_{i h}^{(p)}$ is the number of elements from the $h$-th column of $D_{p}$ which get put into the $i$-th column of $F_{p} / F_{p-1}$.) Note that since $\operatorname{LM}(\tilde{\Delta})=\prod_{j=1}^{k} L_{j}\left(X^{(j)}\right)$, the lemma follows from equation (11).

Now equations (11) and (12) clearly hold for $j=1$. We now assume these equations hold for $1 \leq j \leq m$ where $1 \leq m \leq k-1$ and shall prove that they hold 
for $j=m+1$. Since the proof is similar to that for Proposition 2.4.1, which is given in HTW3, we will only outline the main ideas.

(a) Since we have assumed that equation (12) holds for $j=m, \delta_{T_{1}, \ldots, T_{k-1}}^{(m)}$ is the coefficient of $\prod_{p=m+1}^{k}\left(\beta^{(p)}\right)^{M_{p}}$ in the expansion of the determinant $\operatorname{det} \tilde{X}[m]$.

(b) As in [HTW3, we shall call a column

$$
\left(\begin{array}{c}
\widetilde{X}_{1 h}^{(m+1)}[j] \\
\widetilde{X}_{2 h}^{(m+1)}[j] \\
\cdot \\
\cdot \\
\cdot \\
\widetilde{X}_{t h}^{(m+1)}[j]
\end{array}\right)
$$

of blocks a "supercolumn" of $\widetilde{X}^{(m+1)}[m]$, and a row of blocks

$$
\left(\widetilde{X}_{u 1}^{(m+1)}[j], \widetilde{X}_{u 2}^{(m+1)}[j], \ldots, \widetilde{X}_{u, r_{m+1}}^{(m+1)}[j]\right)
$$

a "superrow" of $\widetilde{X}^{(m+1)}[m]$. We shall also call the lowest nonzero block in each supercolumn the "final block" of that supercolumn.

(c) The form of the matrix $M_{m+1}\left(T_{m}\right)$ implies that $\widetilde{X}^{(m+1)}[m]$ has a block triangular form. More precisely, if the final block of the $h$-th supercolumn occurs in the $i(h)$-th superrow, then $i(h)<i(h+1)$ for all $h$.

(d) We note that the top left entry of the final block of the $h$-th supercolumn is of the form $x_{a_{h}, 1}^{(m+1)}$ and has the highest order among all other entries in the same supercolumn. We call this entry the "reference entry" of the $h$-th supercolumn. Since $L_{m+1}\left(X^{(m+1)}\right)$ has the highest order among all the possible $x^{(m+1)}$-parts which occur in the monomials of $\delta_{T_{1}, \ldots, T_{k-1}}^{(m)}$, it must contain the product of all reference entries as a factor. (To be sure of this, one must show that the sum of all terms divisible by the product of the reference entries is nonzero. This is done in [HTW3.) More precisely, since there are $r_{m+1}$ supercolumns in $\widetilde{X}^{(m+1)}[j], L_{m+1}\left(X^{(m+1)}\right)$ contains the factor

$$
e_{1}^{(m+1)}\left(T_{m}\right)=\prod_{h=1}^{r_{m+1}} x_{a_{h}, 1}^{(m+1)}
$$

(e) Let $\tilde{X}[m] / 1$ be the matrix which is obtained from $\tilde{X}[m]$ by removing all the columns and rows which contain the reference entries $x_{a_{h}, 1}^{(m+1)}$. To determine the variables occurring in $L_{m+1}\left(X^{(m+1)}\right) / e_{1}^{(m+1)}\left(T_{m}\right)$, we consider the coefficient of

$$
\left[\prod_{p=m+1}^{k}\left(\beta^{(p)}\right)^{M_{p}}\right] /\left[\prod_{h=1}^{r_{m+1}} \beta_{i(h), h}^{(m+1)}\right]
$$

in the determinant of $\tilde{X}[m]_{/ 1}$. We denote this function by $\left(\delta_{T_{1}, \ldots, T_{k-1}}^{(m)}\right)_{/ 1}$, and we need to determine the largest $x^{(m+1)}$-part which occurs in the monomials of $\left(\delta_{T_{1}, \ldots, T_{k-1}}^{(m)}\right) / 1$. 
(f) We now compare $e_{1}^{(m+1)}\left(T_{m}\right)$ with $T_{m}$. Recall that for each $1 \leq h \leq$ $r_{m+1}$, the final block in the $h$-th supercolumn occurs in the $i(h)$-th superrow. We note that when the process of standard peeling is reversed, the entry 1 from the $h$-th column of $D_{m+1}$ gets put in the box in the $a_{h}$-th row and the $i(h)$-th column of the skew diagram $F_{m} / F_{m-1}$. Hence the factor $e_{1}^{(m+1)}\left(T_{m}\right)$ divides $e^{(m+1)}\left(T_{m}\right)$, and it accounts for all the boxes in $T_{m}$ filled with a 1 .

(g) Let $\left(T_{m}\right)_{/ 1}$ be the subtableau of $T_{m}$ obtained by omitting all cells of $T_{m}$ which contain 1 . By comparing the tableau $\left(T_{m}\right)_{/ 1}$, the matrix $\widetilde{X}[m]_{/ 1}$ and the monomial

$$
e_{/ 1}^{(m+1)}\left(T_{m}\right)=e^{(m+1)}\left(T_{m}\right) / e_{1}\left(T_{m}\right),
$$

we see that they are parallel to the original triple consisting of the tableau $T_{m}$, the matrix $\tilde{X}[m]$ and the monomial $e^{(m+1)}\left(T_{m}\right)$.

(h) Let $\ell_{0}$ be the largest number which occurs in the cells of $T_{m}$. By repeating the arguments $(\mathrm{d})-(\mathrm{g})$ for $v=2,3, \ldots, \ell_{0}$, we will successively obtain those variables of the form $x_{u v}^{(m+1)}$ which occur in $L_{m+1}\left(X^{(m+1)}\right)$. This proves equation (11) for $j=m+1$. Also, repeating the arguments in (e) proves equation (12) for $j=m+1$.

3.4. Example. We now illustrate the above proof with an example. Let

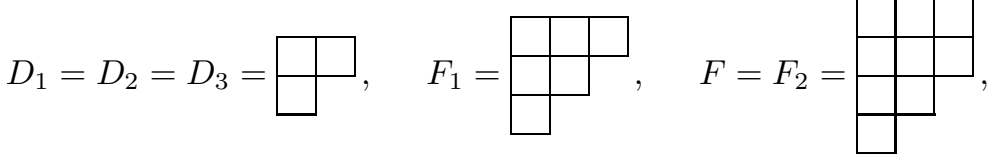

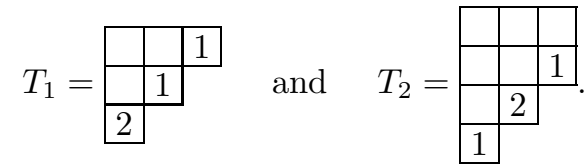

In this case, $\widetilde{X}=$

$\left(\begin{array}{cc|c|cc|c|cc|c}x_{11}^{(1)} & x_{12}^{(1)} & 0 & \beta_{11}^{(2)} x_{11}^{(2)} & \beta_{11}^{(2)} x_{12}^{(2)} & \beta_{12}^{(2)} x_{11}^{(2)} & \beta_{11}^{(3)} x_{11}^{(3)} & \beta_{11}^{(3)} x_{12}^{(3)} & \beta_{12}^{(3)} x_{11}^{(3)} \\ x_{21}^{(1)} & x_{22}^{(1)} & 0 & \beta_{11}^{(2)} x_{21}^{(2)} & \beta_{11}^{(2)} x_{22}^{(2)} & \beta_{12}^{(2)} x_{21}^{(2)} & \beta_{11}^{(3)} x_{21}^{(3)} & \beta_{11}^{(3)} x_{22}^{(3)} & \beta_{12}^{(3)} x_{21}^{(3)} \\ x_{31}^{(1)} & x_{32}^{(1)} & 0 & \beta_{11}^{(2)} x_{31}^{(2)} & \beta_{11}^{(2)} x_{32}^{(2)} & \beta_{12}^{(2)} x_{31}^{(2)} & \beta_{11}^{(3)} x_{31}^{(3)} & \beta_{11}^{(3)} x_{32}^{(3)} & \beta_{12}^{(3)} x_{31}^{(3)} \\ x_{41}^{(1)} & x_{42}^{(1)} & 0 & \beta_{11}^{(2)} x_{41}^{(2)} & \beta_{11}^{(2)} x_{42}^{(2)} & \beta_{12}^{(2)} x_{41}^{(2)} & \beta_{11}^{(3)} x_{41}^{(3)} & \beta_{11}^{(3)} x_{42}^{(3)} & \beta_{12}^{(3)} x_{41}^{(3)} \\ \hline 0 & 0 & x_{11}^{(1)} & \beta_{21}^{(2)} x_{11}^{(2)} & \beta_{21}^{(2)} x_{12}^{(2)} & \beta_{22}^{(2)} x_{11}^{(2)} & \beta_{21}^{(3)} x_{11}^{(3)} & \beta_{21}^{(3)} x_{12}^{(3)} & \beta_{22}^{(3)} x_{11}^{(3)} \\ 0 & 0 & x_{21}^{(1)} & \beta_{21}^{(2)} x_{21}^{(2)} & \beta_{21}^{(2)} x_{22}^{(2)} & \beta_{22}^{(2)} x_{21}^{(2)} & \beta_{21}^{(3)} x_{21}^{(3)} & \beta_{21}^{(3)} x_{22}^{(3)} & \beta_{22}^{(3)} x_{21}^{(3)} \\ 0 & 0 & x_{31}^{(1)} & \beta_{21}^{(2)} x_{31}^{(2)} & \beta_{21}^{(2)} x_{32}^{(2)} & \beta_{22}^{(2)} x_{31}^{(2)} & \beta_{21}^{(3)} x_{31}^{(3)} & \beta_{21}^{(3)} x_{32}^{(3)} & \beta_{22}^{(3)} x_{31}^{(3)} \\ \hline 0 & 0 & 0 & \beta_{31}^{(2)} x_{11}^{(2)} & \beta_{31}^{(2)} x_{12}^{(2)} & \beta_{32}^{(2)} x_{11}^{(2)} & \beta_{31}^{(3)} x_{11}^{(3)} & \beta_{31}^{(3)} x_{12}^{(3)} & \beta_{32}^{(3)} x_{11}^{(3)} \\ 0 & 0 & 0 & \beta_{31}^{(2)} x_{21}^{(2)} & \beta_{31}^{(2)} x_{22}^{(2)} & \beta_{32}^{(2)} x_{21}^{(2)} & \beta_{31}^{(3)} x_{21}^{(3)} & \beta_{31}^{(3)} x_{22}^{(3)} & \beta_{32}^{(3)} x_{21}^{(3)}\end{array}\right)$,

and

$$
M\left(T_{1}, T_{2}\right)=\left(\begin{array}{cc|cc}
m_{11}^{(2)} & m_{12}^{(2)} & m_{11}^{(3)} & m_{12}^{(3)} \\
m_{21}^{(2)} & m_{22}^{(2)} & m_{21}^{(3)} & m_{22}^{(3)} \\
m_{31}^{(2)} & m_{32}^{(2)} & m_{31}^{(3)} & m_{32}^{(3)}
\end{array}\right)=\left(\begin{array}{cc|cc}
1 & 0 & 0 & 1 \\
0 & 1 & 1 & 0 \\
1 & 0 & 1 & 0
\end{array}\right)
$$


So the highest weight vector $\tilde{\Delta}=\tilde{\Delta}_{\left(D_{1}, D_{2}, D_{3}, F\right), M\left(T_{1}, T_{2}\right)}$ is the coefficient of

$$
\beta^{M\left(T_{1}, T_{2}\right)}=\beta_{11}^{(2)} \beta_{31}^{(2)} \beta_{22}^{(2)} \beta_{21}^{(3)} \beta_{31}^{(3)} \beta_{12}^{(3)}
$$

in the expansion of $\operatorname{det} \tilde{X}$. Now the leading monomial of $\tilde{\Delta}$ can be written as

$$
\operatorname{LM}(\tilde{\Delta})=L_{1}\left(X^{(1)}\right) L_{2}\left(X^{(2)}\right) L_{3}\left(X^{(3)}\right),
$$

where $L_{1}\left(X^{(1)}\right), L_{2}\left(X^{(2)}\right)$ and $L_{3}\left(X^{(3)}\right)$ are the $x^{(1)}, x^{(2)}$ and $x^{(3)}$-parts of $\operatorname{LM}(\tilde{\Delta})$, respectively. It is clear that $L_{1}\left(X^{(1)}\right)=x_{11}^{(1)} x_{22}^{(1)} x_{11}^{(1)}$. To determine $L_{2}\left(X^{(2)}\right)$, we consider the sum of all the terms in $\tilde{\Delta}$ which contain $x_{11}^{(1)} x_{22}^{(1)} x_{11}^{(1)}$ as a factor. This sum is given by $x_{11}^{(1)} x_{22}^{(1)} x_{11}^{(1)} \delta_{T_{1}, T_{2}}^{(1)}$, where $\delta_{T_{1}, T_{2}}^{(1)}$ is the coefficient of $\beta^{M\left(T_{1}, T_{2}\right)}$ in $\operatorname{det} \widetilde{X}[1]$, and where

$$
\widetilde{X}[1]=\left(\begin{array}{cc|c|cc|c}
\beta_{11}^{(2)} x_{31}^{(2)} & \beta_{11}^{(2)} x_{32}^{(2)} & 0 & 0 & 0 & \beta_{12}^{(3)} x_{31}^{(3)} \\
\beta_{11}^{(2)} x_{41}^{(2)} & \beta_{11}^{(2)} x_{42}^{(2)} & 0 & 0 & 0 & \beta_{12}^{(3)} x_{41}^{(3)} \\
\hline 0 & 0 & \beta_{22}^{(2)} x_{21}^{(2)} & \beta_{21}^{(3)} x_{21}^{(3)} & \beta_{21}^{(3)} x_{22}^{(3)} & 0 \\
0 & 0 & \beta_{22}^{(2)} x_{31}^{(2)} & \beta_{21}^{(3)} x_{31}^{(3)} & \beta_{21}^{(3)} x_{32}^{(3)} & 0 \\
\hline \beta_{31}^{(2)} x_{11}^{(2)} & \beta_{31}^{(2)} x_{12}^{(2)} & 0 & \beta_{31}^{(3)} x_{11}^{(3)} & \beta_{31}^{(3)} x_{12}^{(3)} & 0 \\
\beta_{31}^{(2)} x_{21}^{(2)} & \beta_{31}^{(2)} x_{22}^{(2)} & 0 & \beta_{31}^{(3)} x_{21}^{(3)} & \beta_{31}^{(3)} x_{22}^{(3)} & 0
\end{array}\right) .
$$

Now the reference entries in $\widetilde{X}^{(2)}[1]$ are $\beta_{31}^{(2)} x_{11}^{(2)}$ and $\beta_{22}^{(2)} x_{21}^{(2)}$, so that $e_{1}^{(2)}\left(T_{1}\right)=$ $x_{11}^{(2)} x_{21}^{(2)}$. To determine the remaining variables in $L_{2}\left(X^{(2)}\right)$ from $\widetilde{X}^{(2)}[1]$, we consider the function $\left(\delta_{T_{1}, T_{2}}^{(1)}\right) / 1$ which is the coefficient of $\beta_{11}^{(2)} \beta_{21}^{(3)} \beta_{31}^{(3)} \beta_{12}^{(3)}$ in

$$
\operatorname{det}\left(\begin{array}{c|cc|c}
\beta_{11}^{(2)} x_{32}^{(2)} & 0 & 0 & \beta_{12}^{(3)} x_{31}^{(3)} \\
\beta_{11}^{(2)} x_{42}^{(2)} & 0 & 0 & \beta_{12}^{(3)} x_{41}^{(3)} \\
\hline 0 & \beta_{21}^{(3)} x_{31}^{(3)} & \beta_{21}^{(3)} x_{32}^{(3)} & 0 \\
\hline \beta_{31}^{(2)} x_{22}^{(2)} & \beta_{31}^{(3)} x_{21}^{(3)} & \beta_{31}^{(3)} x_{22}^{(3)} & 0
\end{array}\right) .
$$

Since the monomial $\beta_{11}^{(2)} \beta_{21}^{(3)} \beta_{31}^{(3)} \beta_{12}^{(3)}$ does not contain $\beta_{31}^{(2)}$, we may replace the $(3,1)$-th block of the above matrix by 0 . In other words, $\left(\delta_{T_{1}, T_{2}}^{(1)}\right) / 1$ is the coefficient of $\beta_{11}^{(2)} \beta_{21}^{(3)} \beta_{31}^{(3)} \beta_{12}^{(3)}$ in

$$
\operatorname{det}\left(\begin{array}{c|cc|c}
\beta_{11}^{(2)} x_{32}^{(2)} & 0 & 0 & \beta_{12}^{(3)} x_{31}^{(3)} \\
\beta_{11}^{(2)} x_{42}^{(2)} & 0 & 0 & \beta_{12}^{(3)} x_{41}^{(3)} \\
\hline 0 & \beta_{21}^{(3)} x_{31}^{(3)} & \beta_{21}^{(3)} x_{32}^{(3)} & 0 \\
\hline 0 & \beta_{31}^{(3)} x_{21}^{(3)} & \beta_{31}^{(3)} x_{22}^{(3)} & 0
\end{array}\right) .
$$

We note that the only reference entry is $\beta_{11}^{(2)} x_{32}^{(2)}$. So $L_{2}\left(X^{(2)}\right)=x_{11}^{(2)} x_{21}^{(2)} x_{32}^{(2)}$, and $\delta_{T_{1}, T_{2}}^{(2)}$ is the coefficient of $\beta_{21}^{(3)} \beta_{31}^{(3)} \beta_{12}^{(3)}$ in the determinant

$$
\operatorname{det}\left(\begin{array}{cc|c}
0 & 0 & \beta_{12}^{(3)} x_{41}^{(3)} \\
\hline \beta_{21}^{(3)} x_{31}^{(3)} & \beta_{21}^{(3)} x_{32}^{(3)} & 0 \\
\hline \beta_{31}^{(3)} x_{21}^{(3)} & \beta_{31}^{(3)} x_{22}^{(3)} & 0
\end{array}\right) .
$$

By repeating the above arguments, we obtain $L_{3}\left(X^{(3)}\right)=x_{21}^{(3)} x_{32}^{(3)} x_{41}^{(3)}$. So the leading monomial of $\tilde{\Delta}$ is given by

$$
\operatorname{LM}(\tilde{\Delta})=\left(x_{11}^{(1)}\right)^{2} x_{22}^{(1)} x_{11}^{(2)} x_{21}^{(2)} x_{32}^{(2)} x_{21}^{(3)} x_{32}^{(3)} x_{41}^{(3)} .
$$


3.5. Main theorem. The following theorem generalizes the results in [HTW3].

Theorem 3.5.1. Let $k \geq 2$ and $\Omega_{k}$ be the set of all $2 k$-tuples

$$
\left(D_{1}, \ldots, D_{k}, F, T_{1}, \ldots, T_{k-1}\right)
$$

where

(i) $D_{1}, . ., D_{k}, F$ are Young diagrams, $r\left(D_{i}\right) \leq l_{i}$ for $1 \leq i \leq k$ and $r(F) \leq$ $n$;

(ii) there are Young diagrams

$$
F_{0}=D_{1}, F_{1}, \ldots, F_{k-2}, F=F_{k-1}
$$

such that for each $1 \leq j \leq k-1, T_{j}$ is an LR tableau of shape $F_{j} / F_{j-1}$ and content $D_{j+1}$.

Then the set

$$
\left\{\tilde{\Delta}_{\left(D_{1}, \ldots, D_{k}, F\right), M\left(T_{1}, \ldots, T_{k-1}\right)}:\left(D_{1}, \ldots, D_{k}, F_{1}, \ldots, F_{k-1}, T_{1}, \ldots, T_{k-1}\right) \in \Omega_{k}\right\}
$$

is a basis for $\mathrm{TA}_{n, l_{1}, \ldots, l_{k}}$.

Proof. By Lemma 3.3.1, the leading term of $\tilde{\Delta}=\tilde{\Delta}_{\left(D_{1}, \ldots, D_{m}, F\right), M\left(T_{1}, \ldots, T_{k-1}\right)}$ is given by

$$
\operatorname{LM}(\tilde{\Delta})=\left[\prod_{j=1}^{r_{1}}\left(\prod_{i=1}^{d_{j}^{(1)}} x_{i i}^{(1)}\right)\right]\left[\prod_{j=1}^{k-1}\left(\prod_{\mathbf{b} \in T_{j}} x_{a_{j}(\mathbf{b}) c_{j}(\mathbf{b})}^{(j+1)}\right)\right] .
$$

We note that it specifies the following information:

(i) For each $1 \leq p \leq k-1$ and each $1 \leq i \leq n$, let $\lambda_{i}^{(p)}$ be the number of entries of the form $x_{i h}^{(j)}$ with $j \leq p+1$ occurring in $\operatorname{LM}(\tilde{\Delta})$ (counting multiplicity). Then

$$
F_{p}=\left(\lambda_{1}^{(p)}, \lambda_{2}^{(p)}, \ldots, \lambda_{n}^{(p)}\right) .
$$

(ii) For each $1 \leq j \leq k$ and $1 \leq h \leq l_{j}$, let $\mu_{h}^{(j)}$ be the number of entries of the form $x_{i h}^{(j)}$ occurring in $\operatorname{LM}(\tilde{\Delta})$ (counting multiplicity). Then

$$
D_{j}=\left(\mu_{1}^{(j)}, \mu_{2}^{(j)}, \ldots, \mu_{l_{j}}^{(j)}\right) .
$$

(iii) For each $1 \leq j \leq k-1$ and $1 \leq i \leq f_{1}^{(j)}$, the numbers occurring in the $i$-th row of $T_{j}$ are given by $c_{j}(\mathbf{b})$, where $\mathbf{b} \in T_{j}$ and $a_{j}(\mathbf{b})=i$ (counting multiplicity), and are arranged in a weakly increasing manner from left to right in $T_{j}$.

From (i) and (ii), we know that the numbers in (iii) are inserted into the skew diagrams $F_{j} / F_{j-1}$. So $T_{j}$ is uniquely determined by $\operatorname{LM}(\tilde{\Delta})$. Hence $\operatorname{LM}(\tilde{\Delta})$ determines $\left(D_{1}, \ldots, D_{k}, F, T_{1}, \ldots, T_{k-1}\right)$. This shows that the assignment

$$
\left(D_{1}, \ldots, D_{k}, F, T_{1}, \ldots, T_{k-1}\right) \rightarrow \tilde{\Delta} \rightarrow \operatorname{LM}(\tilde{\Delta})
$$

is one-to-one. Consequently, the $\tilde{\Delta}$ 's form a linearly independent set. In addition, for fixed $\left(D_{1}, \ldots, D_{k}, F\right)$, the associated LR tableaux $\left(T_{1}, \ldots, T_{k-1}\right)$ count the multiplicity of $\rho_{n}^{F}$ in $\rho_{n}^{D_{1}} \otimes \cdots \otimes \rho_{n}^{D_{k}}$. Hence by varying $\left(T_{1}, \ldots, T_{k-1}\right)$, the $\tilde{\Delta}$ 's form a basis for the $\psi_{n}^{D} \times \psi_{l_{1}}^{D_{1}} \times \cdots \times \psi_{l_{k}}^{D_{k}}$ weight space of $\mathrm{TA}_{n, l_{1}, \ldots, l_{k}}$. By varying the diagrams $D_{1}, \ldots, D_{k}, F$, we obtain a basis for $\mathrm{TA}_{n, l_{1}, \ldots, l_{k}}$. 
4. A BASIS FOR $\mathcal{P}\left(V_{n, k, l}\right)^{U_{n} \times U_{k} \times U_{l}}$

4.1. The algebra $\mathcal{P}\left(V_{n, k, l}\right)$. Let $n, k, l \in \mathbb{Z}^{+}$and let

$$
V_{n, k, l}=\mathrm{M}_{n, k}(\mathbb{C}) \oplus \mathrm{M}_{n l}(\mathbb{C}) .
$$

Let

$$
(X, \hat{Y})=\left(\begin{array}{cccccccccccc}
x_{11} & x_{12} & \cdot & \cdot & \cdot & x_{1 k} & \hat{y}_{11} & \hat{y}_{12} & \cdot & \cdot & \cdot & \hat{y}_{1 l} \\
x_{21} & x_{22} & \cdot & \cdot & \cdot & x_{2 k} & \hat{y}_{21} & \hat{y}_{22} & \cdot & \cdot & \cdot & \hat{y}_{2 l} \\
\cdot & \cdot & & & \cdot & \cdot & \cdot & & & \cdot \\
\cdot & \cdot & & & & \cdot & \cdot & \cdot & & & \cdot \\
x_{n 1} & x_{n 2} & \cdot & \cdot & \cdot & x_{n k} & \hat{y}_{n 1} & \hat{y}_{n 2} & \cdot & \cdot & \cdot & \hat{y}_{n l}
\end{array}\right)
$$

be the system of standard coordinates on $V_{n, k, l}$. We have chosen the awkward notation $\hat{Y}$ and $\hat{y}_{i j}$ because this coordinate system is not convenient for our construction, and we will replace it with a new set of coordinates (using the notation $\left.Y=\left(y_{i j}\right)\right)$ in the next subsection.

Now let the groups $\mathrm{GL}_{n}, \mathrm{GL}_{k}$ and $\mathrm{GL}_{l}$ act on $V_{n, k, l}$ by

$$
\left(g, h_{1}, h_{2}\right)(X, \hat{Y})=\left(\left(g^{-1}\right)^{t} X h_{1}^{-1}, g \hat{Y} h_{2}^{-1}\right),
$$

where $(X, \hat{Y}) \in V_{n, k, l}, g \in \mathrm{GL}_{n}, h_{1} \in \mathrm{GL}_{k}$ and $h_{2} \in \mathrm{GL}_{l}$. Thus as a $\mathrm{GL}_{n} \times \mathrm{GL}_{k} \times$ $\mathrm{GL}_{l}$ module,

$$
V_{n, k, l} \cong\left(\mathbb{C}^{n *} \otimes \mathbb{C}^{k *}\right) \oplus\left(\mathbb{C}^{n} \otimes \mathbb{C}^{l *}\right)
$$

where $\mathbb{C}^{n *}, \mathbb{C}^{k *}$ and $\mathbb{C}^{l *}$ are the contragredient representations of $\mathbb{C}^{n}, \mathbb{C}^{k}$ and $\mathbb{C}^{l}$ respectively. This action induces an action of these groups on the algebra $\mathcal{P}\left(V_{n, k, l}\right)$ of polynomial functions on $V_{n, k, l}$. In this section, we shall construct a basis for the algebra

$$
\mathcal{P}\left(V_{n, k, l}\right)^{U_{n} \times U_{k} \times U_{l}}
$$

of $\mathrm{GL}_{n} \times \mathrm{GL}_{k} \times \mathrm{GL}_{l}$ highest weight vectors in $\mathcal{P}\left(V_{n, k, l}\right)$ in the case when $n \geq k+l$.

So from now on, we shall always assume that $n \geq k+l$. We first consider the $\mathrm{GL}_{n} \times \mathrm{GL}_{k} \times \mathrm{GL}_{l}$ module structure of $\mathcal{P}\left(V_{n, k, l}\right)$. For $1 \leq a \leq k$ and $1 \leq b \leq l$, let

$$
r_{a b}^{2}=\sum_{i=1}^{n} x_{i a} \hat{y}_{i b}
$$

and

$$
\Delta_{a b}=\sum_{i=1}^{n} \frac{\partial^{2}}{\partial x_{i a} \partial \hat{y}_{i b}}
$$

By the first fundamental theorem of invariant theory for $\mathrm{GL}_{n}$ ([Ho95]), the algebra $\mathcal{P}\left(V_{n, k, l}\right)^{\mathrm{GL}_{n}}$ of $\mathrm{GL}_{n}$ invariants is generated by the $r_{a b}^{2}$ 's. Since $n \geq k+l$, there are no nontrivial relations among these generators. Consequently,

$$
\begin{aligned}
\mathcal{P}\left(V_{n, k, l}\right)^{\mathrm{GL}_{n}} & =\mathbb{C}\left[r_{a b}^{2}: 1 \leq a \leq k, 1 \leq b \leq l\right] \\
& \cong \mathcal{P}\left(\mathrm{M}_{k l}(\mathbb{C})\right) .
\end{aligned}
$$

Moreover, under the action of $\mathrm{GL}_{k} \times \mathrm{GL}_{l}$,

$$
\mathcal{P}\left(V_{n, k, l}\right)^{\mathrm{GL}} \cong \sum_{r(F) \leq \min (k, l)} \rho_{k}^{F} \otimes \rho_{l}^{F} .
$$

On the other hand, let

$$
\mathcal{H}\left(V_{n, k, l}\right)=\left\{f \in \mathcal{P}\left(V_{n, k, l}\right): \Delta_{a b}(f)=0,1 \leq a \leq k, 1 \leq b \leq l\right\} .
$$


Under the action of $\mathrm{GL}_{n} \times \mathrm{GL}_{k} \times \mathrm{GL}_{l}$,

$$
\mathcal{H}\left(V_{n, k, l}\right) \cong \sum_{\substack{r(D) \leq k \\ r(E) \leq l}} \rho_{n}^{D, E} \otimes \rho_{k}^{D} \otimes \rho_{l}^{E} .
$$

The condition $n \geq k+l$ implies that ([Ho95]

$$
\mathcal{P}\left(V_{n, k, l}\right)=\mathcal{H}\left(V_{n, k, l}\right) \otimes \mathcal{P}\left(V_{n, k, l}\right)^{\mathrm{GL}_{n}} .
$$

It follows that under the action of $\mathrm{GL}_{n} \times \mathrm{GL}_{k} \times \mathrm{GL}_{l}$,

$$
\begin{aligned}
\mathcal{P}\left(V_{n, k, l}\right) & =\left(\sum_{\substack{r(D) \leq k \\
r(E) \leq l}} \rho_{n}^{D, E} \otimes \rho_{k}^{D} \otimes \rho_{l}^{E}\right) \otimes\left(\sum_{r(F) \leq \min (k, l)} \rho_{k}^{F} \otimes \rho_{l}^{F}\right) \\
& =\sum_{\substack{r(D) \leq k, r(E) \leq l \\
r(F) \leq \min (k, l)}} \rho_{n}^{D, E} \otimes\left(\rho_{k}^{D} \otimes \rho_{k}^{F}\right) \otimes\left(\rho_{l}^{E} \otimes \rho_{l}^{F}\right) \\
& \sum_{\substack{r(D) \leq k, r(E) \leq l \\
r(F) \leq \min (k, l)}} \rho_{n}^{D, E} \otimes\left(\sum_{G} c_{D, F}^{G} \rho_{k}^{G}\right) \otimes\left(\sum_{H} c_{E, F}^{H} \rho_{l}^{H}\right) \\
& =\sum_{\substack{r(D) \leq k, r(E) \leq l \\
G, H}}\left(\sum_{r(F) \leq \min (k, l)} c_{D, F}^{G} c_{E, F}^{H}\right) \rho_{n}^{D, E} \otimes \rho_{k}^{G} \otimes \rho_{l}^{H}
\end{aligned}
$$

by the Littlewood-Richardson rule. Taking $U_{n} \times U_{k} \times U_{l}$ invariants, we obtain

$$
\begin{aligned}
& \mathcal{P}\left(V_{n, k, l}\right)^{U_{n} \times U_{k} \times U_{l}} \\
& \cong \sum_{\substack{r(D) \leq k, r(E) \leq l \\
G, H}}\left(\sum_{r(F) \leq \min (k, l)} c_{D, F}^{G} c_{E, F}^{H}\right)\left(\rho_{n}^{D, E} \otimes \rho_{k}^{G} \otimes \rho_{l}^{H}\right)^{U_{n} \times U_{k} \times U_{l}} .
\end{aligned}
$$

Note that by the theory of highest weights,

$$
\operatorname{dim}\left(\rho_{n}^{D, E} \otimes \rho_{k}^{G} \otimes \rho_{l}^{H}\right)^{U_{n} \times U_{k} \times U_{l}}=\operatorname{dim}\left[\left(\rho_{n}^{D, E}\right)^{U_{n}} \otimes\left(\rho_{k}^{G}\right)^{U_{k}} \otimes\left(\rho_{l}^{H}\right)^{U_{l}}\right]=1 .
$$

Thus if $\tau=\rho_{n}^{D, E} \otimes \rho_{k}^{G} \otimes \rho_{l}^{H}$ and $\mathcal{P}\left(V_{n, k, l}\right)_{\tau}$ is the $\tau$-isotypic component in $\mathcal{P}\left(V_{n, k, l}\right)$, then

$$
\begin{aligned}
\operatorname{dim}\left[\mathcal{P}\left(V_{n, k, l}\right)_{\tau}\right]^{U_{n} \times U_{k} \times U_{l}} & =\operatorname{dim}\left[\mathcal{P}\left(V_{n, k, l}\right)^{U_{n} \times U_{k} \times U_{l}} \cap \mathcal{P}\left(V_{n, k, l}\right)_{\tau}\right] \\
& =\sum_{r(F) \leq \min (k, l)} c_{D, F}^{G} c_{E, F}^{H} .
\end{aligned}
$$

4.2. Highest weight vectors in $\mathcal{P}\left(V_{n, k, l}\right)$. We now fix four Young diagrams $D, E$, $G$ and $H$ with $r(D), r(G) \leq k$ and $r(E), r(H) \leq l$, and let $\tau=\rho_{n}^{D, E} \otimes \rho_{k}^{G} \otimes \rho_{l}^{H}$. By equation (13), a basis of $\left[\mathcal{P}\left(V_{n, k, l}\right)_{\tau}\right]^{U_{n} \times U_{k} \times U_{l}}$ consists of $\sum_{r(F) \leq \min (k, l)} c_{D, F}^{G} c_{E, F}^{H}$ elements. So we shall also fix a Young diagram $F$ such that $c_{D, F}^{G} c_{E, F}^{H} \neq 0$. Note that $c_{D, F}^{G} c_{E, F}^{H}$ is equal to the number of ordered pairs $\left(T_{1}, T_{2}\right)$ of LR tableaux with the following properties:

(i) $T_{1}$ is of shape $G / F$ and content $D$,

(ii) $T_{2}$ is of shape $H / F$ and content $E$. 
We shall associate each such ordered pair $\left(T_{1}, T_{2}\right)$ with a $\mathrm{GL}_{n} \times \mathrm{GL}_{k} \times \mathrm{GL}_{l}$ highest weight vector $\Delta$ of weight $\psi_{n}^{D, E} \times \psi_{k}^{G} \times \psi_{l}^{H}$. Eventually, we shall prove that the set $\{\Delta\}$ of highest weight vectors obtained in this way forms a basis for $\mathcal{P}\left(V_{n, k, l}\right)^{U_{n} \times U_{k} \times U_{l}}$.

We now set up some notation. Write

$$
\begin{aligned}
D^{t} & =\left(d_{1}, d_{2}, \ldots, d_{r}\right), \\
E^{t} & =\left(e_{1}, e_{2}, \ldots, e_{s}\right), \\
F^{t} & =\left(f_{1}, f_{2}, \ldots, f_{t}\right), \\
G^{t} & =\left(g_{1}, g_{2}, \ldots, g_{u}\right), \\
H^{t} & =\left(h_{1}, h_{2}, \ldots, h_{v}\right) .
\end{aligned}
$$

Thus the components are the column lengths of the diagrams $D, E, F, G$ and $H$. The standard coordinates $\hat{y}=\left(\hat{y}_{i j}\right)$ on $\mathrm{M}_{n, l}(\mathbb{C})$ are not convenient in our construction of the highest weight vector $\Delta$. We shall replace them with $Y=\left(y_{i j}\right)$, where

$$
y_{i j}=\hat{y}_{n+1-i, j} \quad(1 \leq i \leq n, 1 \leq j \leq l) .
$$

Let $\alpha=\left(\alpha_{i j}\right), \beta=\left(\beta_{a b}\right), \gamma=\left(\gamma_{m}\right)$ be matrices of indeterminates of size $u \times r$, $v \times s$ and $1 \times t$ respectively. For $1 \leq i \leq u, 1 \leq j \leq r, 1 \leq a \leq v, 1 \leq b \leq s$ and $1 \leq m \leq t$,

$$
\begin{aligned}
& \widetilde{X}_{i j}=\alpha_{i j}\left(\begin{array}{cccccc}
x_{11} & x_{21} & \cdot & \cdot & \cdot & x_{d_{j}, 1} \\
x_{12} & x_{22} & \cdot & \cdot & \cdot & x_{d_{j}, 2} \\
\cdot & \cdot & & & & \cdot \\
\cdot & \cdot & & & & \cdot \\
\cdot & \cdot & & & & \cdot \\
x_{1, g_{i}} & x_{2, g_{i}} & \cdot & \cdot & \cdot & x_{d_{j}, g_{i}}
\end{array}\right) \text {, } \\
& \widetilde{Y}_{a b}=\beta_{a b}\left(\begin{array}{cccccc}
y_{11} & y_{12} & \cdot & \cdot & \cdot & y_{1, h_{a}} \\
y_{21} & y_{22} & \cdot & \cdot & \cdot & y_{2, h_{a}} \\
\cdot & \cdot & & & & \cdot \\
\cdot & \cdot & & & \cdot \\
\cdot & \cdot & & & \cdot \\
y_{e_{b}, 1} & y_{e_{b}, 2} & \cdot & \cdot & \cdot & y_{e_{b}, h_{a}}
\end{array}\right) \text {, } \\
& \widetilde{R}_{m}=\gamma_{m}\left(\begin{array}{cccc}
r_{11}^{2} & r_{12}^{2} & \cdots & r_{1, h_{m}}^{2} \\
r_{21}^{2} & r_{22}^{2} & \cdots & r_{2, h_{m}}^{2} \\
\cdot & \cdot & & \cdot \\
\cdot & \cdot & & \cdot \\
\cdot & \cdot & & \cdot \\
r_{g_{m}, 1}^{2} & r_{g_{m}, 2}^{2} & \cdots & r_{g_{m}, h_{m}}^{2}
\end{array}\right) .
\end{aligned}
$$

We now combine all these matrices to form a single matrix $\widetilde{Z}$ as follows: $\widetilde{Z}$ is a square matrix with size

$$
(|E|+|G|) \times(|D|+|H|)=(|E|+|G|) \times(|E|+|G|),
$$

and its rows and columns are partitioned according to

$$
\left(e_{1}, e_{2}, \ldots, e_{s}, g_{1}, g_{2}, \ldots, g_{u}\right)
$$

and

$$
\left(d_{1}, d_{2}, \ldots, d_{r}, h_{1}, h_{2}, \ldots, h_{v}\right)
$$


respectively. In block matrix form,

$$
\widetilde{Z}=\left(\begin{array}{cccc|ccccc}
0 & 0 & \cdots & 0 & \widetilde{Y}_{11} & \widetilde{Y}_{21} & \widetilde{Y}_{31} & \cdots & \widetilde{Y}_{v 1} \\
0 & 0 & \cdots & 0 & \widetilde{Y}_{12} & \widetilde{Y}_{22} & \widetilde{Y}_{32} & \cdots & \widetilde{Y}_{v 2} \\
\cdot & \cdot & & \cdot & \cdot & \cdot & \cdot & & \cdot \\
\cdot & \cdot & & \cdot & \cdot & \cdot & \cdot & & \cdot \\
\cdot & \cdot & & \cdot & \cdot & \cdot & \cdot & & \cdot \\
0 & 0 & \cdots & 0 & \widetilde{Y}_{1 s} & \widetilde{Y}_{2 s} & \widetilde{Y}_{3 s} & \cdots & \widetilde{Y}_{v s} \\
\hline & & & & & & & & \\
\hline \widetilde{X}_{11} & \widetilde{X}_{12} & \cdots & \widetilde{X}_{1 r} & \widetilde{R}_{1} & 0 & 0 & \cdots & 0 \\
\widetilde{X}_{21} & \widetilde{X}_{22} & \cdots & \widetilde{X}_{2 r} & 0 & \widetilde{R}_{2} & 0 & \cdots & 0 \\
\widetilde{X}_{31} & \widetilde{X}_{32} & \cdots & \widetilde{X}_{3 r} & 0 & 0 & \widetilde{R}_{3} & \cdots & 0 \\
\cdot & \cdot & & \cdot & \cdot & \cdot & \cdot & & \cdot \\
\cdot & \cdot & & \cdot & \cdot & \cdot & \cdot & & \cdot \\
\cdot & \cdot & & \cdot & \cdot & \cdot & \cdot & & \cdot \\
\widetilde{X}_{u 1} & \widetilde{X}_{u 2} & \cdots & \widetilde{X}_{u r} & 0 & 0 & 0 & \cdots &
\end{array}\right) .
$$

We now let

$$
\Delta_{(D, E, G, H),(\alpha, \beta, \gamma)}=\operatorname{det} \widetilde{Z}
$$

Lemma 4.2.1. $\Delta=\Delta_{(D, E, G, H),(\alpha, \beta, \gamma)}$ is a $\mathrm{GL}_{n} \times \mathrm{GL}_{k} \times \mathrm{GL}_{l}$ highest weight vector with weight $\psi_{n}^{D, E} \times \psi_{k}^{G} \times \psi_{l}^{H}$.

Proof. The weight of $\Delta$ can be verified easily. This is left to the readers. We shall only show that $\Delta$ is annihilated by the infinitesimal generators of the groups $U_{n}$, $U_{k}$ and $U_{l}$.

We first consider $U_{n}$. It has a basis of infinitesimal generators acting by the operators

$$
\begin{aligned}
E_{i j} & =\sum_{a=1}^{k}\left(x_{i a} \frac{\partial}{\partial x_{j a}}-\hat{y}_{j a} \frac{\partial}{\partial \hat{y}_{i a}}\right) \\
& =\sum_{a=1}^{k}\left(x_{i a} \frac{\partial}{\partial x_{j a}}-y_{n-j+1, a} \frac{\partial}{\partial y_{n-i+1, a}}\right)
\end{aligned}
$$

for $1 \leq i<j \leq n$. Write

$$
\widetilde{X}=\left(\begin{array}{cccccc}
\widetilde{X}_{11} & \widetilde{X}_{12} & \cdot & \cdot & \cdot & \widetilde{X}_{1 r} \\
\widetilde{X}_{21} & \widetilde{X}_{22} & \cdot & \cdot & \cdot & \widetilde{X}_{2 r} \\
\cdot & \cdot & & & \cdot \\
\cdot & \cdot & & & \cdot \\
\cdot & \cdot & & & \cdot \\
\tilde{X}_{u 1} & \tilde{X}_{u 2} & \cdot & \cdot & \cdot & \tilde{X}_{u r}
\end{array}\right), \quad \widetilde{Y}=\left(\begin{array}{cccccc}
\widetilde{Y}_{11} & \widetilde{Y}_{21} & \cdot & \cdot & \cdot & \widetilde{Y}_{v 1} \\
\widetilde{Y}_{12} & \widetilde{Y}_{22} & \cdot & \cdot & \cdot & \widetilde{X}_{v 2} \\
\cdot & \cdot & & & \cdot \\
\cdot & \cdot & & & \cdot \\
\cdot & \cdot & & & \cdot \\
\tilde{Y}_{1 s} & \tilde{Y}_{2 s} & \cdot & \cdot & \cdot & \tilde{Y}_{v s}
\end{array}\right)
$$


and

$$
\widetilde{R}=\left(\begin{array}{ccccccc}
\widetilde{R}_{1} & 0 & 0 & \cdot & \cdot & \cdot & 0 \\
0 & \widetilde{R}_{2} & 0 & \cdot & \cdot & \cdot & 0 \\
0 & 0 & \widetilde{R}_{3} & \cdot & \cdot & \cdot & 0 \\
\cdot & \cdot & \cdot & & & & \cdot \\
\cdot & \cdot & \cdot & & & & \cdot \\
. & \cdot & \cdot & & & & \cdot \\
0 & 0 & 0 & . & \cdot & . &
\end{array}\right) .
$$

Then

$$
\Delta=\operatorname{det}\left(\begin{array}{cc}
0 & \widetilde{Y} \\
\widetilde{X} & \widetilde{R}
\end{array}\right) .
$$

For each $1 \leq a \leq|D|$ and $1 \leq b \leq|E|$, let

$$
\begin{aligned}
\tilde{X}(a)= & \text { the matrix obtained from } \tilde{X} \text { by replacing } \\
& \text { its } a \text {-th column by its image under } E_{i j}, \\
\tilde{Y}(b)= & \text { the matrix obtained from } \tilde{Y} \text { by replacing } \\
& \text { its } b \text {-th row by its image under } E_{i j} .
\end{aligned}
$$

Since $E_{i j}$ is a derivation on $\mathcal{P}\left(V_{n, k, l}\right)$ and $E_{i j}\left(r_{c d}^{2}\right)=0$ for all $1 \leq c \leq k$ and $1 \leq d \leq l$, it can be checked that

$$
E_{i j}(\Delta)=\sum_{a=1}^{|D|} \operatorname{det}\left(\begin{array}{cc}
0 & \widetilde{Y} \\
\widetilde{X}(a) & \widetilde{R}
\end{array}\right)+\sum_{b=1}^{|E|} \operatorname{det}\left(\begin{array}{cc}
0 & \widetilde{Y}(b) \\
\widetilde{X} & \widetilde{R}
\end{array}\right) .
$$

Under the action of $E_{i j}$, the columns of $\widetilde{X}$ are either replaced by the zero column, or if the column of $\widetilde{X}$ contains entries of the form $\alpha_{c d} x_{j e}$, then it gets replaced by a column with entries of the form $\alpha_{c d} x_{i e}$. In the latter case, since $i<j, \widetilde{X}(a)$ contains two repeated columns. Consequently,

$$
\operatorname{det}\left(\begin{array}{cc}
0 & \widetilde{Y} \\
\widetilde{X}(a) & \widetilde{R}
\end{array}\right)=0
$$

for all $1 \leq a \leq|D|$. Similarly,

$$
\operatorname{det}\left(\begin{array}{cc}
0 & \widetilde{Y}(b) \\
\widetilde{X} & \widetilde{R}
\end{array}\right)=0
$$

for all $1 \leq b \leq|E|$. Hence $E_{i j}(\Delta)=0$.

Next we consider the action of a basis of the infinitesimal generators of $U_{k}$ :

$$
L_{i j}=\sum_{a=1}^{n} x_{a i} \frac{\partial}{\partial x_{a j}}, \quad 1 \leq i<j \leq k .
$$

For $1 \leq a \leq|G|$, let

$$
\begin{aligned}
\widetilde{X}_{H}(a)= & \text { the matrix obtained from } \widetilde{X} \text { by replacing } \\
& \text { its } a \text {-th row by its image under } L_{i j}, \\
\widetilde{R}_{H}(a)= & \text { the matrix obtained from } \widetilde{R} \text { by replacing } \\
& \text { its } a \text {-th row by its image under } L_{i j} .
\end{aligned}
$$


Then

$$
L_{i j}(\Delta)=\sum_{a=1}^{|G|} \operatorname{det}\left(\begin{array}{cc}
0 & \widetilde{Y} \\
\widetilde{X}_{H}(a) & \widetilde{R}_{H}(a)
\end{array}\right) .
$$

Now observe that if a row of $\widetilde{X}$ contains only entries of the form $\alpha_{c d} x_{e f}$, then the entries of the same row of $\widetilde{R}$ are either 0 or equal to $\gamma_{c} r_{f h}^{2}$. On the other hand, we note that

$$
L_{i j}\left(r_{u v}^{2}\right)=\left\{\begin{array}{cc}
r_{i v}^{2} & \text { if } u=j, \\
0 & \text { if } u \neq j .
\end{array}\right.
$$

Thus under the action of $L_{i j}$, either both rows are replaced by the zero row, or are replaced by a repeated row in the matrix. Consequently,

$$
\operatorname{det}\left(\begin{array}{cc}
0 & \widetilde{Y} \\
\widetilde{X}_{H}(a) & \widetilde{R}_{H}(a)
\end{array}\right)=0
$$

for $1 \leq a \leq|G|$, and so $L_{i j}(\Delta)=0$. The proof that $\Delta$ is a $\mathrm{GL}_{l}$ highest weight vector is similar.

Now observe that $\Delta_{(D, E, G, H),(\alpha, \beta, \gamma)}$ can be expressed in the form

$$
\Delta_{(D, E, G, H),(\alpha, \beta, \gamma)}=\sum_{M, N, L} \tilde{\Delta}_{(D, E, G, H),(M, N, L)} \alpha^{M} \beta^{N} \gamma^{L},
$$

where $M=\left(m_{i j}\right), N=\left(n_{a b}\right)$ and $L=\left(l_{c}\right)$ are matrices of nonnegative integers and

$$
\alpha^{M}=\prod_{i, j} \alpha_{i j}^{m_{i j}}, \quad \beta^{N}=\prod_{a, b} \beta_{a b}^{n_{a b}} \quad \gamma^{L}=\prod_{c} \gamma_{c}^{l_{c}},
$$

and $\tilde{\Delta}_{(D, E, G, H),(M, N, L)}=\tilde{\Delta}_{(D, E, G, H),(M, N, L)}(X, Y)$ is a polynomial in the variables $X=\left(x_{s t}\right)$ and $Y=\left(y_{u v}\right)$. Recall that $T_{1}$ and $T_{2}$ are LR tableaux of shape $G / F$ and $H / F$ and content $D$ and $E$ respectively. Define $M\left(T_{1}\right)=\left(m_{a b}\right)$ and $N\left(T_{2}\right)=\left(n_{c d}\right)$ and $L(F)$ by

$$
\begin{aligned}
m_{a b}= & \text { the number of elements from the } b \text {-th column of } D \\
& \text { which get put into the } a \text {-th column of } G / F, \\
n_{c d}= & \text { the number of elements from the } d \text {-th column of } E \\
& \text { which get put into the } c \text {-th column of } H / F, \\
L(F)= & F^{t}=\left(f_{1}, f_{2}, \ldots, f_{t}\right) .
\end{aligned}
$$

We consider the $\mathrm{GL}_{n} \times \mathrm{GL}_{k} \times \mathrm{GL}_{l}$ highest weight vector

$$
\tilde{\Delta}_{(D, E, G, H),\left(M\left(T_{1}\right), N\left(T_{2}\right), L(F)\right)}
$$

with weight $\psi_{n}^{D, E} \times \psi_{k}^{G} \times \psi_{l}^{H}$.

Theorem 4.2.2. Let $\Omega$ be the set of 7-tuples

$$
\left(D, E, F, G, H, T_{1}, T_{2}\right)
$$

where

(i) $D, E, F, G$ and $H$ are Young diagrams with $r(D) \leq k, r(E) \leq l$, $r(F) \leq \min (k, l), r(G) \leq k$ and $r(H) \leq l$;

(ii) $T_{1}$ and $T_{2}$ are LR tableaux of shape $G / F$ and $H / F$ and content $D$ and E respectively. 
Then the set

$$
\left\{\tilde{\Delta}_{(D, E, G, H),\left(M\left(T_{1}\right), N\left(T_{2}\right), L(F)\right)}:\left(D, E, F, G, H, T_{1}, T_{2}\right) \in \Omega\right\}
$$

forms a basis for $\mathcal{P}\left(V_{n, k, l}\right)^{U_{n} \times U_{k} \times U_{l}}$.

We shall prove this theorem in Sections 4.3 to 4.5.

4.3. Algebraically independent variables in $\mathcal{P}\left(V_{n, k, l}\right)$. We note that the subset

$$
A=\left\{x_{i j}, y_{a b}, r_{c d}^{2}: 1 \leq i, j, c \leq k, 1 \leq a, b, d \leq l\right\}
$$

of $\mathcal{P}\left(V_{n, k, l}\right)$ is algebraically independent. In fact, since $n \geq k+l$, we have an isomorphism of $\mathrm{GL}_{n} \times \mathrm{GL}_{k} \times \mathrm{GL}_{l}$ modules

$$
\mathcal{P}\left(V_{n, k, l}\right) \cong \mathcal{H}\left(V_{n, k, l}\right) \otimes \mathcal{P}\left(V_{n, k, l}\right)^{\mathrm{GL}_{n}} .
$$

Now

$$
\begin{aligned}
\mathcal{P}\left(\mathrm{M}_{k, k}(\mathbb{C}) \oplus \mathrm{M}_{l, l}(\mathbb{C})\right) & \cong \mathbb{C}\left[x_{i j}, y_{a b}: 1 \leq i, j \leq k, 1 \leq a, b \leq l\right] \\
& \subseteq \mathcal{H}\left(V_{n, k, l}\right) .
\end{aligned}
$$

Hence the subalgebra of $\mathcal{P}\left(V_{n, k, l}\right)$ generated by $A$ is isomorphic to

$$
\begin{aligned}
& \mathbb{C}\left[x_{i j}, y_{a b}: 1 \leq i, j \leq k, 1 \leq a, b \leq l\right] \otimes \mathbb{C}\left[r_{c d}^{2}: 1 \leq c \leq k, 1 \leq d \leq l\right] \\
\cong & \mathcal{P}\left(\mathrm{M}_{k, k}(\mathbb{C}) \oplus \mathrm{M}_{l, l}(\mathbb{C})\right) \otimes \mathcal{P}\left(\mathrm{M}_{k, l}(\mathbb{C})\right) ;
\end{aligned}
$$

i.e., this subalgebra is a polynomial algebra on $A$ and $A$ is algebraically independent. We shall denote this subalgebra by $\mathbb{C}[A]$. From now on, $X$ and $Y$ will mean

$$
X=\left(\begin{array}{cccccc}
x_{11} & x_{12} & \cdot & \cdot & \cdot & x_{1 k} \\
x_{21} & x_{22} & \cdot & \cdot & \cdot & x_{2 k} \\
\cdot & \cdot & & & \cdot \\
\cdot & \cdot & & & \cdot \\
\cdot & \cdot & & & \cdot \\
x_{k 1} & x_{k 2} & \cdot & \cdot & \cdot & x_{k k}
\end{array}\right) \quad \text { and } \quad Y=\left(\begin{array}{cccccc}
y_{11} & y_{12} & \cdot & \cdot & \cdot & y_{1 l} \\
y_{21} & y_{22} & \cdot & \cdot & \cdot & y_{2 l} \\
\cdot & \cdot & & & \cdot \\
\cdot & \cdot & & & \cdot \\
\cdot & \cdot & & & \cdot \\
y_{l 1} & y_{l 2} & \cdot & \cdot & \cdot & y_{l l}
\end{array}\right) \text {. }
$$

(In Section 4.1 $X$ and $Y$ were matrices of variables of size $n \times k$ and $n \times l$ respectively.) We also let

$$
R=\left(\begin{array}{cccccc}
r_{11}^{2} & r_{12}^{2} & \cdot & \cdot & \cdot & r_{1 l}^{2} \\
r_{21}^{2} & r_{22}^{2} & \cdot & \cdot & \cdot & r_{2 l}^{2} \\
\cdot & \cdot & & & \cdot \\
\cdot & \cdot & & & \cdot \\
\cdot & \cdot & & & \cdot \\
r_{k 1}^{2} & r_{k 2}^{2} & \cdot & \cdot & \cdot & r_{k l}^{2}
\end{array}\right)
$$

We shall write a polynomial in $\mathbb{C}[A]$ as $p=p(X, Y, R)$. In particular, since $\tilde{\Delta}=$ $\tilde{\Delta}_{(D, E, G, H),\left(M\left(T_{1}\right), N\left(T_{2}\right), L(F)\right)} \in \mathbb{C}[A]$, we shall write $\tilde{\Delta}=\tilde{\Delta}(X, Y, R)$.

4.4. The leading monomial of $\tilde{\Delta}_{(D, E, G, H),\left(M\left(T_{1}\right), N\left(T_{2}\right), L(F)\right)}$. We define an ordering on the monomials in $A$ as follows:

(O1) For all $a, b, c, d, e$ and $f$,

$$
r_{a b}^{2}>x_{c d}>y_{e f},
$$


and

$$
\begin{aligned}
x_{i j}>x_{u v} & \Longleftrightarrow i<u \text {, or } i=u \text { and } j<v, \\
y_{i j}>y_{u v} & \Longleftrightarrow i<u, \text { or } i=u \text { and } j<v, \\
r_{i j}^{2}>r_{u v}^{2} & \Longleftrightarrow i<u \text {, or } i=u \text { and } j<v .
\end{aligned}
$$

(O2) We extend (O1) to an ordering between all monomials in $A$ by the graded lexicographical order ( CLO $)$.

(a) If $x^{M_{1}} y^{N_{1}}\left(r^{2}\right)^{L_{1}}$ has a higher total degree than $x^{M_{2}} y^{N_{2}}\left(r^{2}\right)^{L_{2}}$, then

$$
x^{M_{1}} y^{N_{1}}\left(r^{2}\right)^{L_{1}}>x^{M_{2}} y^{N_{2}}\left(r^{2}\right)^{L_{2}} .
$$

(b) If $x^{M_{1}} y^{N_{1}}\left(r^{2}\right)^{L_{1}}$ and $x^{M_{2}} y^{N_{2}}\left(r^{2}\right)^{L_{2}}$ have the same total degree, then we shall arrange the variables which occur in these monomials in decreasing order. The larger monomial is the one with higher first nonequal variable.

Lemma 4.4.1. The leading monomial $\operatorname{LM}(\tilde{\Delta})$ of $\tilde{\Delta}=\tilde{\Delta}_{(D, E, G, H),\left(M\left(T_{1}\right), N\left(T_{2}\right), L(F)\right)}$ with respect to the monomial ordering (O1)-(O2) is given by

$$
\operatorname{LM}(\tilde{\Delta})=\left(\prod_{\mathbf{b}_{1} \in T_{1}} x_{c_{1}\left(\mathbf{b}_{1}\right) a_{1}\left(\mathbf{b}_{1}\right)}\right)\left(\prod_{\mathbf{b}_{2} \in T_{2}} y_{c_{2}\left(\mathbf{b}_{2}\right) a_{2}\left(\mathbf{b}_{2}\right)}\right)\left[\prod_{i=1}^{t}\left(\prod_{j=1}^{f_{i}} r_{j j}^{2}\right)\right],
$$

where

(i) $a_{1}\left(\mathbf{b}_{1}\right)$ is the row of $G / D$ in which the box $\mathbf{b}_{1}$ lies, and $c_{1}\left(\mathbf{b}_{1}\right)$ is the entry in $\mathbf{b}_{1}$, and

(ii) $a_{2}\left(\mathbf{b}_{2}\right)$ is the row of $H / E$ in which the box $\mathbf{b}_{2}$ lies, and $c_{2}\left(\mathbf{b}_{2}\right)$ is the entry in $\mathbf{b}_{2}$.

Proof. We recall from equations (18) that $\tilde{\Delta}$ is the coefficient of the monomial

$$
\alpha^{M\left(T_{1}\right)} \beta^{N\left(T_{2}\right)} \gamma^{L(F)}
$$

in the polynomial $\operatorname{det} \widetilde{Z}$ given in equation (14), where the matrices $M\left(T_{1}\right), N\left(T_{2}\right)$ and $L(F)$ are defined in equations (15), (16) and (17) respectively. This means that $\tilde{\Delta}$ is a sum of the terms in the expansion of $\operatorname{det} \widetilde{Z}$ which satisfy the condition that $m_{i j}$ of the entries are taken from $\widetilde{X}_{i j}, n_{a b}$ from $\widetilde{Y}_{a b}$ and $f_{m}$ from $\widetilde{R}_{m}$. From the definition of the monomial ordering, it is clear that $\operatorname{LM}(\tilde{\Delta})$ contains the factor

$$
w_{F}(R)=\prod_{i=1}^{t}\left(\prod_{j=1}^{f_{i}} r_{j j}^{2}\right)
$$

Let $\delta_{T_{1}, T_{2}}$ be the coefficient of $w_{F}(R)$ in $\tilde{\Delta}$; that is, $\delta_{T_{1}, T_{2}}$ is the sum of all the terms of $\tilde{\Delta}$ that contain $w_{F}(R)$ as a factor. We note that since $w_{F}$ has already exhausted the entries which are needed from $\widetilde{R}, \delta_{T_{1}, T_{2}}$ is a polynomial in $\left\{x_{a b}, y_{c d}\right.$ : $1 \leq a, b \leq k, 1 \leq c, d \leq l\}$, and $\operatorname{LM}(\tilde{\Delta})$ is the product of the leading monomial in $\delta_{T_{1}, T_{2}}$ and $w_{F}(R)$. Thus it suffices to compute the leading monomial of $\delta_{T_{1}, T_{2}}$. 
To determine the explicit form of $\delta_{T_{1}, T_{2}}$, we need to delete the rows and columns of $\widetilde{Z}$ containing the variables $\gamma_{i} r_{11}^{2}, \gamma_{i} r_{22}^{2}, \ldots, \gamma_{i} r_{f_{i}, f_{i}}^{2}$ for $1 \leq i \leq t$. Thus we define the following submatrices of $\widetilde{X}$ and $\tilde{Y}$ :

(i) $\widetilde{X}_{0}$ is $|D| \times|D|$ and is divided into $u r$ blocks. Its $(i, j)$-th block is given by

$$
\left(\widetilde{X}_{0}\right)_{i j}=\alpha_{i j}\left(\begin{array}{cccccc}
x_{1, f_{i}+1} & x_{2, f_{i}+1} & \cdot & \cdot & \cdot & x_{d_{j}, f_{i}+1} \\
x_{1, f_{i}+2} & x_{2, f_{i}+2} & \cdot & \cdot & \cdot & x_{d_{j}, f_{i}+2} \\
\cdot & \cdot & & & & \cdot \\
\cdot & \cdot & & & \cdot \\
\cdot & \cdot & & & & \cdot \\
x_{1, g_{i}} & x_{2, g_{i}} & \cdot & \cdot & \cdot & x_{d_{j}, g_{i}}
\end{array}\right) .
$$

(ii) $\widetilde{Y}_{0}$ is $|E| \times|E|$ and is divided into $v s$ blocks. Its $(a, b)$-th block is given by

$$
\left(\widetilde{Y}_{0}\right)_{a b}=\beta_{a b}\left(\begin{array}{cccccc}
y_{1, f_{i}+1} & y_{1, f_{i}+2} & \cdot & \cdot & \cdot & y_{1, h_{a}} \\
y_{2, f_{i}+1} & y_{2, f_{i}+2} & \cdot & \cdot & \cdot & y_{2, h_{a}} \\
\cdot & \cdot & & & & \cdot \\
\cdot & \cdot & & & \cdot \\
\cdot & \cdot & & & \\
y_{e_{b}, f_{i}+1} & y_{e_{b}, f_{i}+2} & \cdot & \cdot & \cdot & y_{e_{b}, h_{a}}
\end{array}\right) .
$$

Since $\widetilde{X}_{0}$ and $\widetilde{Y}_{0}$ are square matrices, we can take their determinants. The determinants can be expressed in the form

$$
\begin{aligned}
\operatorname{det} \tilde{X}_{0} & =\sum_{M} p_{M}(X) \alpha^{M}, \\
\operatorname{det} \widetilde{Y}_{0} & =\sum_{N} q_{N}(Y) \beta^{N} .
\end{aligned}
$$

It is clear that

$$
\delta_{T_{1}, T_{2}}(X, Y)= \pm\left[p_{M\left(T_{1}\right)}(X)\right]\left[q_{N\left(T_{2}\right)}(Y)\right]
$$

We now recall that in Section 2.4, we have defined a polynomial $\delta_{\left(D^{\prime}, E^{\prime}, F^{\prime}\right), T^{\prime}}$ for any LR tableau $T^{\prime}$ of shape $F^{\prime} / D^{\prime}$ and content $E^{\prime}$. We now consider $\delta_{T_{1}}=\delta_{(F, D, G), T_{1}}$ and $\delta_{T_{2}}=\delta_{(F, E, H), T_{2}}$. Note that $\delta_{T_{1}}$ is a polynomial on $\mathrm{M}_{k q}(\mathbb{C})$ where $q=r(D)$. So it can be identified with a polynomial in $X \in \mathrm{M}_{k k}(\mathbb{C})$. Similarly, $\delta_{T_{2}}$ can be identified with a polynomial in $Y \in \mathrm{M}_{l l}(\mathbb{C})$. It is easy to see that $p_{M\left(T_{1}\right)}(X)=$ $\delta_{T_{1}}\left(X^{t}\right)$ and $q_{N\left(T_{2}\right)}(Y)=\delta_{T_{2}}\left(Y^{t}\right)$, so that

$$
\delta_{T_{1}, T_{2}}(X, Y)= \pm\left[\delta_{T_{1}}\left(X^{t}\right)\right]\left[\delta_{T_{2}}\left(Y^{t}\right)\right]
$$

Here $X^{t}$ and $Y^{t}$ denote the transposes of the matrices $X$ and $Y$. Moreover, the monomial ordering in $\mathbb{C}[A]$ restricts to the monomial ordering defined in Section 2.4 for $\mathbb{C}\left[x_{i j}: 1 \leq i, j \leq k\right]$ and $\mathbb{C}\left[y_{a b}: 1 \leq a, b \leq l\right]$. Hence the expression for $\operatorname{LM}(\tilde{\Delta})$ now follows from Proposition 2.4.1.

4.5. Proof of Theorem 4.2.2. The $\mathrm{GL}_{n} \times \mathrm{GL}_{k} \times \mathrm{GL}_{l}$ weight of $\tilde{\Delta}$ determines the diagrams $D, E, G$ and $H$. Consider the leading monomial $\operatorname{LM}(\tilde{\Delta})$ of $\tilde{\Delta}$ given in Lemma 4.4.1. We note that its $R$-part determines the diagram $F$, its $X$-part and $Y$-part determine the numbers occurring in the rows of the tableaux $T_{1}$ and 
$T_{2}$ respectively. The shapes of $T_{1}$ and $T_{2}$ are $G / F$ and $H / F$, which are already determined. So $T_{1}$ and $T_{2}$ are determined. This shows that

$$
\left(D, E, F, G, H, T_{1}, T_{2}\right) \rightarrow \tilde{\Delta} \rightarrow \operatorname{LM}(\tilde{\Delta})
$$

is one-to-one. So the $\tilde{\Delta}$ 's are linearly independent. By varying $D, E, G, H, T_{1}$ and $T_{2}$, the $\tilde{\Delta}$ 's form a basis for $\mathcal{P}\left(V_{n, k, l}\right)^{U_{n} \times U_{k} \times U_{l}}$.

4.6. Example. Let

$$
D=E=F=\square, \quad G=H=\begin{aligned}
& \square \\
& \square
\end{aligned} .
$$

In this case, $\widetilde{Z}=$

$\left(\begin{array}{cc|c|ccc|cc|c}0 & 0 & 0 & \beta_{11} y_{11} & \beta_{11} y_{12} & \beta_{11} y_{13} & \beta_{21} y_{11} & \beta_{21} y_{12} & \beta_{31} y_{11} \\ 0 & 0 & 0 & \beta_{11} y_{21} & \beta_{11} y_{22} & \beta_{11} y_{23} & \beta_{21} y_{21} & \beta_{21} y_{22} & \beta_{31} y_{21} \\ \hline 0 & 0 & 0 & \beta_{12} y_{11} & \beta_{12} y_{12} & \beta_{12} y_{13} & \beta_{22} y_{11} & \beta_{22} y_{12} & \beta_{32} y_{11} \\ \hline \alpha_{11} x_{11} & \alpha_{11} x_{21} & \alpha_{12} x_{11} & \gamma_{1} r_{11}^{2} & \gamma_{1} r_{12}^{2} & \gamma_{1} r_{13}^{2} & 0 & 0 & 0 \\ \alpha_{11} x_{12} & \alpha_{11} x_{22} & \alpha_{12} x_{12} & \gamma_{1} r_{21}^{2} & \gamma_{1} r_{22}^{2} & \gamma_{1} r_{23}^{2} & 0 & 0 & 0 \\ \alpha_{11} x_{13} & \alpha_{11} x_{23} & \alpha_{12} x_{13} & \gamma_{1} r_{31}^{2} & \gamma_{1} r_{32}^{2} & \gamma_{1} r_{33}^{2} & 0 & 0 & 0 \\ \hline \alpha_{21} x_{11} & \alpha_{21} x_{21} & \alpha_{22} x_{11} & 0 & 0 & 0 & \gamma_{2} r_{11}^{2} & \gamma_{2} r_{12}^{2} & 0 \\ \alpha_{21} x_{12} & \alpha_{21} x_{22} & \alpha_{22} x_{12} & 0 & 0 & 0 & \gamma_{2} r_{21}^{2} & \gamma_{2} r_{22}^{2} & 0 \\ \hline \alpha_{31} x_{11} & \alpha_{31} x_{21} & \alpha_{32} x_{11} & 0 & 0 & 0 & 0 & 0 & 0\end{array}\right)$.

There are four choices for the pair $\left(T_{1}, T_{2}\right)$ of LR tableaux. Here we consider two cases.

(i) If

$$
T_{1}=T_{2}=\begin{array}{|l|l|l|}
\hline & & 1 \\
\hline & 1 & \\
\hline 2 &
\end{array},
$$

then

$$
M\left(T_{1}\right)=N\left(T_{2}\right)=\left(\begin{array}{ll}
1 & 0 \\
0 & 1 \\
1 & 0
\end{array}\right) .
$$

So $\tilde{\Delta}$ is the coefficient of

$$
\alpha_{11} \alpha_{22} \alpha_{31} \beta_{11} \beta_{22} \beta_{31} \gamma_{1}^{2} \gamma_{2}
$$

in $\operatorname{det} \widetilde{Z}$, and its leading monomial is given by

$$
\operatorname{LM}(\tilde{\Delta})=x_{11} x_{12} x_{23} y_{11} y_{12} y_{23}\left(r_{11}^{2}\right)^{2} r_{22}^{2}
$$

(ii) If

$$
T_{1}=\begin{array}{|l|l|l|}
\hline & & 1 \\
\hline & 1
\end{array} \quad \text { and } \quad T_{2}=\begin{array}{|l|l|l|}
\hline & & 1 \\
\hline 2 & & 2 \\
\hline 1
\end{array}
$$

then

$$
M\left(T_{1}\right)=\left(\begin{array}{ll}
1 & 0 \\
0 & 1 \\
1 & 0
\end{array}\right) \quad \text { and } \quad N\left(T_{2}\right)=\left(\begin{array}{ll}
0 & 1 \\
1 & 0 \\
1 & 0
\end{array}\right) .
$$

So $\tilde{\Delta}$ is the coefficient of

$$
\alpha_{11} \alpha_{22} \alpha_{31} \beta_{12} \beta_{21} \beta_{31} \gamma_{1}^{2} \gamma_{2}
$$


in $\operatorname{det} \widetilde{Z}$, and its leading monomial is given by

$$
\operatorname{LM}(\tilde{\Delta})=x_{11} x_{12} x_{23} y_{11} y_{22} y_{13}\left(r_{11}^{2}\right)^{2} r_{22}^{2} \text {. }
$$

4.7. Highest weight module for $\mathfrak{u}(k, l)_{\mathbb{C}}$. From the theory of compact dual pairs ([Ho89, [KV] $), \mathcal{P}\left(V_{n, k, l}\right)$ admits a decomposition

$$
\mathcal{P}\left(V_{n, k, l}\right) \cong \sum_{r(D) \leq k, r(E) \leq l} \rho_{n}^{D, E} \otimes W_{k, l}^{D, E},
$$

where $\rho_{n}^{D, E}$ is the irreducible representation of $\mathrm{GL}_{n}$ associated with $(D, E)$, and $W_{k, l}^{D, E}$ is a highest weight module for $\mathfrak{u}(k, l)_{\mathbb{C}} \cong \mathfrak{g} l_{k+l}(\mathbb{C})$. Then

$$
\mathcal{P}\left(V_{n, k, l}\right)^{U_{n} \times U_{k} \times U_{l}} \cong \sum_{D, E}\left(\rho_{n}^{D, E}\right)^{U_{n}} \otimes\left(W_{k, l}^{D, E}\right)^{U_{k} \times U_{l}} .
$$

For fixed Young diagrams $D_{0}$ and $E_{0}$, since $\operatorname{dim}\left(\rho_{n}^{D_{0}, E_{0}}\right)^{U_{n}}=1$, we can identify $W_{k, l}^{D_{0}, E_{0}}$ with the subspace $\left(\rho_{n}^{D_{0}, E_{0}}\right)^{U_{n}} \otimes W_{k, l}^{D_{0}, E_{0}}$ in $\mathcal{P}\left(V_{n, k, l}\right)$. The following proposition is now clear.

Proposition 4.7.1. Let $D_{0}$ and $E_{0}$ be Young diagrams with $r\left(D_{0}\right) \leq k$ and $r\left(E_{0}\right) \leq l$, and identify $W_{k, l}^{D_{0}, E_{0}}$ with the subspace $\left(\rho_{n}^{D, E}\right)^{U_{n}} \otimes\left(W_{k, l}^{D, E}\right)^{\bar{U}_{k} \times U_{l}}$ in $\mathcal{P}\left(V_{n, k, l}\right)^{U_{k} \times U_{l}}$. Then the set

$$
\left\{\Delta_{\left(D_{0}, E_{0}, G, H\right),\left(M\left(T_{1}\right), N\left(T_{2}\right), L(F)\right)}:\left(D_{0}, E_{0}, F, G, H, T_{1}, T_{2}\right) \in \Omega\right\}
$$

is a basis for the space of all $\mathfrak{g} l_{k}(\mathbb{C}) \oplus \mathfrak{g} l_{l}(\mathbb{C})$ highest weight vectors in $W_{k, l}^{D_{0}, E_{0}}$.

\section{ACKNOWLEDGMents}

The second named author expresses his sincere gratitude to Yale University for warm hospitality during his visit in Fall, 2003.

\section{REFERENCES}

[BBL] G. Benkart, D. Britten and F. Lemire, Stability in modules for classical Lie algebras: A constructive approach, Mem. Amer. Math. Soc. 85 (1990), no. 430. MR1010997 (90m:17012)

[BL] M. Brion and V. Lakshmibai, A geometric approach to standard monomial theory, Represent. Theory 7 (2003), 651-680. MR2017071(2004m:14106)

[BZ] A. Berenstein and A. Zelevinsky, Triple multiplicities for $\mathfrak{s l}(r+1)$ and the spectrum of the exterior algebra of the adjoint representation, J. Algebraic Combin. 1 (1992), no. 1, 7-22. MR:1162639 (93h:17012)

[Ca] P. Caldero, Toric degenerations of Schubert Varieties, Transform. Groups 7 (2002), no. 1, 51-60. MR1888475 (2003a:14073)

[Ch] R. Chirivi, LS algebras and application to Schubert varieties Transform. Groups 5 (2000), no. 3, 245-264. MR1780934 (2001h:14060)

[CLO] D. Cox, J. Little and D. O'Shea, Ideals, Varieties, and Algorithms. An introduction to Computational Algebraic Geometry and Commutative Algebra, Second edition, Undergraduate Texts in Mathematics, Springer-Verlag, New York, 1997. MR1417938 (97h:13024)

[DEP] C. De Concini, D. Eisenbud and C. Procesi, Hodge algebras, Astérisque, 91. Société Mathématique de France, Paris, 1982. MR0680936 (85d:13009)

[EHW] T. Enright, R. Howe, and N. Wallach, A classification of unitary highest weight modules, in Representation Theory of Reductive Groups (P.C. Trombi, Ed.), pp. 97 - 143, Progr. Math., 40, Birkhäuser, Boston, MA, 1983. MR0733809 (86c:22028) 
[Ful] W. Fulton, Young Tableaux, London Mathematical Society Student Texts 35, Cambridge University Press, Cambridge, UK, 1997. MR.1464693 (99f:05119)

[GL] N. Gonciulea and V. Lakshmibai, Degenerations of flag and Schubert varieties to toric varieties, Transform. Groups 1 (1996), no. 3, 215-248. MR1417711 (98a:14065)

[GW] R. Goodman and N. R. Wallach, Representations and Invariants of the Classical Groups, Encyclopedia of Mathematics and its Applications 68. Cambridge University Press, Cambridge, 1998. MR1606831 (99b:20073)

[Hd] W.V.D. Hodge, Some enumerative results in the theory of forms, Proc. Cambridge Philos. Soc. 39 (1943), 22 - 30. MR0007739 (4:184e)

[Ho83] R. Howe, Reciprocity laws in the theory of dual pairs, in Representation Theory of Reductive Groups, Prog. in Math. 40, P. Trombi, ed., Birkhaüser, Boston (1983), 159 175. MR0733812 (85k:22033)

[Ho89] R. Howe, Remarks on classical invariant theory, Trans. Amer. Math. Soc. 313 (1989), 539 - 570. MR0986027 (90h:22015a)

[Ho95] R. Howe, Perspectives on Invariant Theory, The Schur Lectures, I. Piatetski-Shapiro and S. Gelbart (eds.), Israel Mathematical Conference Proceedings, 1995, 1 - 182. MR $1321638(96 \mathrm{e}: 13006)$

[HTW1] R. Howe, E-C. Tan and J. Willenbring, Reciprocity Algebras and Branching for Classical Symmetric Pairs. arXiv:math.RT/0407467

[HTW2] R. Howe, E-C. Tan and J. Willenbring, Stable Branching Rules for Classical Symmetric Pairs, Trans. Amer. Math. Soc. 357 (2005), 1601-1626. MR2115378 (2005j:22007)

[HTW3] R. Howe, E-C. Tan and J. Willenbring, A Basis for the GL $L_{n}$ Tensor Product Algebra, Adv. Math. 196 (2005), 531-564. MR2166314 (2006h:20062)

[KM] M. Kogan and E. Miller, Toric degeneration of Schubert varieties and Gelfand-Cetlin polytopes. Adv. Math. 193 (2005), 1-17. MR2132758 (2006d:14054)

[Kud] S. Kudla, Seesaw dual reductive pairs, in Automorphic Forms of Several Variables, Taniguchi Symposium, Katata, 1983, Birkhäuser, Boston (1983), 244 - 268. MR0763017 (86b:22032)

[KV] M. Kashiwara and M. Vergne, On the Segal-Shale-Weil representations and harmonic polynomials, Invent. Math. 44 (1978), 1 - 47. MR0463359 (57:3311)

[L1] V. Lakshmibai, Geometry of G/P. VI. Bases for fundamental representations of classical groups, J. Algebra 108 (1987), no. 2, 355-402. MR0892910 (88i:14046a)

[L2] V. Lakshmibai, Geometry of G/P. VII. The symplectic group and the involution $\sigma$, J. Algebra 108 (1987), no. 2, 403-434. MR0892911 (88i:14046b)

[L3] V. Lakshmibai, Geometry of G/P. VIII. The groups $\mathrm{SO}(2 n+1)$ and the involution $\sigma$, J. Algebra 108 (1987), no. 2, 435-471. MR0892912 (88i:14046c)

[L4] V. Lakshmibai, Standard monomial theory for $\widehat{\mathrm{SL}}_{n}$ in Operator algebras, unitary representations, enveloping algebras, and invariant theory (Paris, 1989), 197-217, Progr. Math., 92, Birkhäuser Boston, Boston, MA, 1990. MR1103591 (92f:14052)

[LLM] V. Lakshmibai, P. Littelmann and P. Magyar, Standard monomial theory and applications, Notes by Rupert W. T. Yu, NATO Adv. Sci. Inst. Ser. C Math. Phys. Sci., 514, Representation theories and algebraic geometry (Montreal, PQ, 1997), Kluwer Acad. Publ., Dordrecht, 1998, 319 - 364. MR 1653037 (99j:20050)

[LMS1] V. Lakshmibai, C. Musili and C.S. Seshadri, Geometry of G/P. III. Standard monomial theory for a quasi-minuscule P, Proc. Indian Acad. Sci. Sect. A Math. Sci. 88 (1979), no. 3, 93-177. MR0561813 (81g:14023c)

[LMS2] V. Lakshmibai, C. Musili and C.S. Seshadri, Geometry of G/P. IV. Standard monomial theory for classical types, Proc. Indian Acad. Sci. Sect. A Math. Sci. 88 (1979), no. 4, 279-362. MR0553746 (81g:14023d)

[LS] V. Lakshmibai and C. S. Seshadri, Standard monomial theory, Proceedings of the Hyderabad Conference on Algebraic Groups (Hyderabad, 1989), Manoj Prakashan, Madras, 1991, 279 - 322. MR1131317 (92k:14053)

[Mac] I. Macdonald, Symmetric Functions and Hall Polynomials, Clarendon Press, Oxford, 1979. MR0553598 (84g:05003)

[RS] L. Rubbiano and M. Sweedler, Subalgebra bases, in Commutative algebra (Salvador, 1988), Lecture Notes in Math., 1430, Springer, Berlin, 1990, 61 - 87. MR1068324 (91f:13027) 
[Stu] B. Sturmfels, Gröbner Bases and Convex Polytopes, Univ. Lecture Series, Vol. 8, Amer. Math. Soc., Providence, RI, 1996. MR1363949 (97b:13034)

[Wey] H. Weyl, The Classical Groups, Princeton Univ. Press, Princeton, 1946. MR 1488158 (98k:01049)

[Zhe] D. Zhelobenko, Compact Lie Groups and their Representations, Transl. of Math. Mono. 40, AMS, Providence, R.I., 1973. MR0473098 (57:12776b)

Department of Mathematics, Yale University, New Haven, Connecticut 06520-8283

E-mail address: howe@math.yale.edu

Department of Mathematics, National University of Singapore, 2 Science Drive 2, SINGAPORE 117543

E-mail address: matleest@nus.edu.sg 